\title{
¿ENSO Diversity in a Tropical Stochastic Skeleton Model for the MJO, EI Niño, and Dynamic Walker Circulation
}

\author{
QiU YANG, ${ }^{a}$ ANDREW J. MAJDA, ${ }^{\mathrm{a}, \mathrm{b}}$ AND NAN CHEN ${ }^{\mathrm{c}}$ \\ ${ }^{a}$ Center for Prototype Climate Modeling, New York University Abu Dhabi, Saadiyat Island, Abu Dhabi, United Arab Emirates \\ ${ }^{\mathrm{b}}$ Department of Mathematics and Center for Atmosphere Ocean Science, Courant Institute of Mathematical Sciences, \\ New York University, New York, New York \\ ${ }^{\mathrm{c}}$ Department of Mathematics, University of Wisconsin-Madison, Madison, Wisconsin
}

(Manuscript received 10 June 2020, in final form 22 December 2020)

\begin{abstract}
El Niño-Southern Oscillation (ENSO) diversity has a significant impact on global climate and seasonal prediction. However, it is still a challenging problem for present-day global climate models to simulate different types of ENSO events with realistic features simultaneously. In this paper, a tropical stochastic skeleton model for the interactions among wind bursts and the Madden-Julian oscillation (MJO), the El Niño, and the Walker circulation is developed to reproduce both dynamical and statistical features of the ENSO diversity. In this model, the intraseasonal component with state-dependent noise captures general features of wind bursts and the MJO, both of which play important roles in triggering the El Niño. The thermocline feedback is the dominant mechanism for generating the eastern Pacific (EP) El Niño, while a nonlinear zonal advection is incorporated into the model that contributes to the central Pacific (CP) El Niño. Besides, a simple but effective stochastic process describing the multidecadal variation of the background Walker circulation modulates the spatial patterns and occurrence frequency of the EP and CP El Niño. This model succeeds in simulating the quasi-regular moderate EP El Niño, the super El Niño, and the CP El Niño as well as the La Niña simultaneously. It also captures the observed non-Gaussian characteristics of sea surface temperature anomalies in different Niño regions. Individual case studies highlight the outstanding skill of the model in reproducing the observed El Niño episodes and their underlying mechanisms.
\end{abstract}

KEYWORDS: Tropical variability; ENSO; Madden-Julian oscillation; Walker circulation; Wind bursts; Stochastic models

\section{Introduction}

ENSO is the dominant interannual atmosphere-ocean coupled system in the tropics, significantly influencing global climate conditions, extreme weather events, agriculture, and ecosystems through teleconnections (Dai and Wigley 2000; McPhaden et al. 2006a; Yeh et al. 2018). Although event-to-event differences have long been observed, it is only in the last two decades that the significance of the ENSO diversity was acknowledged by the scientific community (Kirtman 2019). Besides the eastern Pacific (EP) El Niño events, the observation in the last two decades indicates the increasing occurrence of the central Pacific (CP) El Niño events with warm sea surface temperature (SST) anomalies near the date line (Ashok et al. 2007; Freund et al. 2019). In addition, the ENSO is observed to interact with other physical processes from intraseasonal to interannual to multidecadal scales. For example, the ENSO has significant modulation effects on the MJO (Tam and Lau 2005), and it is also strongly influenced by the multidecadal oscillations (Dong et al. 2006; Gao et al. 2019; Cai et al. 2019) and climate change (Knutson et al. 1997; Cai et al. 2014; Stevenson et al. 2019).

Early ENSO studies date back to Bjerknes (1969) who realized that the ENSO should be a coupled system between the

¿ Denotes content that is immediately available upon publication as open access.

Corresponding author: Qiu Yang, yangq@cims.nyu.edu equatorial Pacific Ocean and atmosphere. Later on, many atmosphere-ocean coupled theories were proposed to explain the ENSO, such as the delayed oscillator theory (Schopf 1987; Schopf and Suarez 1988; Suarez and Schopf 1988; Battisti 1988; Battisti and Hirst 1989), the discharge-recharge oscillator theory (Jin 1997a,b) and the intermediate coupled model (Cane and Zebiak 1985; Zebiak 1986; Zebiak and Cane 1987; Zebiak 1989). Note that these theories aimed at explaining the overall oscillation mechanism instead of capturing the ENSO irregularity and diversity as observed in nature (Wang and Picaut 2004). In fact, it is only in the last 15 years that the scientific community acknowledged the importance of wind bursts for the ENSO mechanisms. For example, Seiki and Takayabu (2007a,b) found the statistical and energetic relationship among westerly wind bursts (WWBs), the ENSO, and the MJO based on the 40-yr reanalysis datasets. Lian et al. (2014) and Chen et al. (2015) further confirmed the effect of WWBs on the ENSO diversity through numerical simulations. Besides, Hu and Fedorov (2016, 2019) highlighted the important role of both westerly and easterly wind bursts in inducing the delayed extreme El Niño in 2014-16. As a major source of wind bursts, the MJO is also shown to interact with the ENSO (McPhaden et al. 2006b; Hendon et al. 2007; Tang and Yu 2008). More importantly, the stochastic nature of wind bursts helps explain the irregular spatiotemporal patterns of the ENSO (Puy et al. 2019a).

Due to the improvement of computing resources, physical parameterization schemes, and data assimilation techniques, present-day global climate models (GCMs) can capture basic features of the ENSO with a 6-month lead prediction skill (Jin 
et al. 2008; Barnston et al. 2012; Tang et al. 2018). However, they still suffer from significant biases in reproducing the ENSO diversity, including the irregularity of the El Niño, the extreme ENSO events (Capotondi et al. 2015), the amplitude asymmetry between El Niño and La Niña phases (An et al. 2005; Zhang and Sun 2014), and the strengths and locations of the CP El Niño events. For example, the delayed super El Niño event in 2014-16 exerted remarkable global influence, but many state-of-the-art GCMs failed to predict and even reproduce it (L. Chen et al. 2017). In addition, large statistical biases in ENSO behaviors are identified in phase 5 of the Climate Model Intercomparison Project (CMIP5) simulations (C. Chen et al. 2017). It has been shown that the poorly simulated ENSO diversity in GCMs is related to the inadequate treatment of the interactions among wind bursts and the MJO, the El Niño, and the Walker circulation (Bellenger et al. 2014; Taschetto et al. 2014) as well as the ocean zonal advection. Here we aim at developing a simple model with mathematical tractability to capture these complex interactions across multiple time scales and providing useful guidelines for improving the ENSO simulations in GCMs.

Recently, a simple stochastic modeling framework has been developed (Thual et al. 2016; Chen and Majda 2016, 2017), which successfully captures the ENSO diversity and the observed non-Gaussian statistics in different equatorial Pacific regions. In this modeling framework, a simple ocean-atmosphere model is augmented by a simple stochastic parameterization of the wind bursts (Thual et al. 2016), the nonlinear zonal advection of SST, a mean easterly trade wind anomaly (Chen and Majda 2016), and a three-state stochastic Markov jump process with prescribed transition rates for regime switching (Chen and Majda 2017). It can capture the ENSO synchronization to the seasonal cycle (Thual et al. 2017) and the formation mechanisms of both the EP and CP El Niño (Chen et al. 2018) and explain the delayed super El Niño in 2014-16 (Thual et al. 2019). To incorporate refined atmospheric wind burst structures into the model and study the interaction between the MJO and the ENSO, Thual et al. (2018) coupled the MJO skeleton model to the ENSO model and developed the first simplified GCM that qualitatively captures major intraseasonal to interannual processes altogether. The MJO skeleton model was first developed by Majda and Stechmann (2009), which explicitly resolves the MJO and wind bursts on the intraseasonal scale. In particular, its stochastic version can account for the intermittent initialization, propagation, termination of the MJO (Thual et al. 2014) and those asymmetric intraseasonal events due to the seasonal cycle (Thual et al. 2015). Note that despite a quite realistic MJO-ENSO coupling, the observed non-Gaussian SST statistics and the occurrence of the CP events have not been captured in this MJOENSO coupled model.

The goals of this study include the following three aspects: first, to incorporate suitable nonlinear mechanisms and statedependent noise to realistically simulate the coupled dynamics of the MJO and wind bursts, the ENSO, and the background Walker circulation; second, to introduce the dynamics of the CP El Niño and reproduce the ENSO diversity with all different types of ENSO events occurring in a realistic frequency; third, to capture the observed non-Gaussian features and irregular spatiotemporal patterns of SST anomalies in different
Niño regions. Despite its simplicity, this model should be the first simple stochastic model that captures many observed features as in nature, including the MJO-ENSO interaction, the El Niño diversity, the non-Gaussian statistics with extreme El Niños and the dynamic Walker circulation. This model may serve to provide useful guidelines for improving ENSO simulations in GCMs.

To achieve the above goals, the following new essential physical mechanisms are added into the tropical stochastic skeleton model (Thual et al. 2018). First, Seiki and Takayabu (2007a,b) showed that the ENSO atmospheric and sea surface conditions influence the occurrence frequency and magnitude of wind bursts, which in turn have a crucial impact on the ENSO dynamics. Thus, we add a multiplicative noise in the intraseasonal skeleton model to mimic these state-dependent wind bursts. Second, we add the nonlinear zonal advection term in the SST budget by following Chen and Majda (2016), which showed that the nonlinear zonal advection of SST along with a background easterly trade wind anomaly plays a key role in triggering $\mathrm{CP}$ El Niño events. Last, previous studies discovered that the multidecadal variation in the background state modulates the spatial patterns and occurrence frequency of the EP and CP El Niño as well as their teleconnection impact on global climate (Freund et al. 2019; Lemmon and Karnauskas 2019; Stevenson et al. 2019; Gao et al. 2019; Cai et al. 2019). To this end, a simple but effective stochastic process is developed to drive the multidecadal variability of the background Walker circulation (L'Heureux et al. 2013). Thus in this model, wind bursts, the ENSO fields, and the Walker circulation are adjusted simultaneously, which is different from the prescribed switching rates in the earlier models (Chen and Majda 2017; Thual et al. 2017; Chen et al. 2018; Thual et al. 2019).

With these new mechanisms and novel stochastic parameterizations, the new coupled model successfully reproduces the ENSO diversity, the non-Gaussian features and realistic occurrence frequency of SST anomalies in different Niño regions, and the observed El Niño episodes. In particular, both EP and CP El Niño events are simulated simultaneously with considerable irregularity in amplitude, duration, temporal evolution, and spatial structure. Also, the equilibrium probability density function (PDF) of SST compares well with the observation in all three Niño regions, including a perfect match of the variance in all Niño-3, Niño-3.4, and Niño-4 regions and good recovery of the skewness direction and kurtosis in Niño-3 and Niño-3.4 ones. Besides, the frequency spectra of SST in these three Niño regions all have a dominant peak within the 2-7-yr band, resembling the observation. Furthermore, the model reproduces diverse types of El Niño episodes, including the delayed super El Niño in 2014-16, the CP El Niño in 2009-10, the 5-yr CP El Niño followed by a super El Niño in the 1990s, and the super El Niño in 1982-83. Last, the model realistically captures the interactions among the $\mathrm{MJO}$ and wind bursts, the ENSO, and the background Walker circulation from intraseasonal to interannual to multidecadal scales.

The rest of the paper is organized as follows. Section 2 summarizes all the details about the governing equations, prescribed profiles of external forcing and other background fields, and the observational SST data. Section 3 shows the 
non-Gaussian features in wind bursts and the MJO. Section 4 investigates the ENSO diversity in the presence of the dynamic Walker circulation. Specifically, subsection 4 a focuses on the scenario with only EP El Niño events, while subsection 4 b considers the one with only CP El Niño events. In subsection 4c, the full model allows the switching between EP and CP regimes and captures the ENSO diversity. The model output is compared with the observation in terms of the equilibrium PDF and frequency spectrum of SST anomalies as well as individual case studies. The paper concludes with a discussion in section 5 .

\section{Model and observational data}

In this section, we first describe the properties of the model that we used in this study. Particular attention has been paid to the new mechanisms that are incorporated into the governing equations. Then we illustrate the profiles of multiple fields such as the external forcing of moisture and cooling as well as background equivalent potential temperature, according to the observation. Last, we summarize the details of the observational SST data that we used for comparison in later sections.

\section{a. Governing equations}

The full model developed here starts with the tropical stochastic skeleton (TSS) model of Thual et al. (2018). The TSS model was developed to capture qualitatively tropical atmosphere-ocean coupling among the MJO and the associated wind bursts, the El Niño, and the background dynamic Walker circulation. It successfully captures the most salient features of the ENSO, the wind bursts, and the MJO, including their overall structure, evolution, and fundamental interactions in addition to their intermittency, diversity, and energy distribution across scales. Yet, this starting model lacks the mechanisms of generating non-Gaussian SST statistics, the CP El Niño, and the ENSO diversity. The mechanisms for explaining all these crucial observational features are incorporated into the full model developed here.

This full model is derived systematically from the primitive equations to model planetary-scale tropical dynamics on intraseasonal, interannual and multidecadal scales. All governing equations are resolved on the zonal planetary-scale $x$ of $15000 \mathrm{~km}$ and intraseasonal scale $t$ of 3.3 days, while the prognostic equations for interannual dynamics have a small factor $\varepsilon=0.1$ to resolve interannual scale of 33 days. Due to different Rossby deformation radius, the meridional coordinate in the atmosphere $y$ is scaled by $1500 \mathrm{~km}$, while that in the ocean $Y$ is scaled by $330 \mathrm{~km}$. As for physical fields, the intraseasonal model only considers atmospheric flows with zonal and meridional winds $u^{\prime}, v^{\prime}$ scaled by 5.0 and $0.5 \mathrm{~m} \mathrm{~s}^{-1}$, potential temperature $\theta^{\prime}$ and moisture $q^{\prime}$ anomaly scaled by $1.5 \mathrm{~K}$ as well as nondimensional envelope magnitude of synoptic convective activity magnitude $a^{\prime}$. The scalings of interannual atmospheric flows are the same as intraseasonal ones. One nondimensional unit of external source of cooling $s^{\theta}$ and moistening $s^{q}$ corresponds to $0.45 \mathrm{~K} \mathrm{day}^{-1}$. Besides, the interannual model considers oceanic flows with zonal and meridional currents $U, V$ scaled by 0.25 and $0.0056 \mathrm{~m} \mathrm{~s}^{-1}$, and thermocline depth anomaly $H$ scaled by $20.8 \mathrm{~m}$ as well as SST anomaly $T$ scaled by $1.5 \mathrm{~K}$. More details about the model derivation and variable scaling can be found in the appendix of Thual et al. (2016) and Chen and Majda $(2016,2017)$ and the context of Thual et al. (2018).

As shown by Table 1, the full model consists of three groups of governing equations, one for intraseasonal atmospheric dynamics of wind bursts and the MJO, one for interannual atmosphere-ocean coupled systems, the third for multidecadal variation of the Walker circulation. The intraseasonal atmosphere model is the skeleton model for the MJO (Majda and Stechmann 2009), where the first, second, and third equations describe the planetary/intraseasonal tropical circulation and the fourth and fifth equations introduce the fundamental mechanism involving neutrally stable interactions between planetary-scale lower-tropospheric moisture anomaly $q^{\prime}$ and the envelope of synoptic-scale convective activity $a^{\prime}$. In contrast, the governing equations for interannual atmosphere are steady (no time derivative) and undamped so that the remaining terms are directly balanced with external sources of cooling $s^{\theta}$ and moistening $s^{q}$ as well as evaporation $E_{q}$. The governing equations for the interannual ocean are under the longwave approximation and forced by wind stress $c_{1} \tau_{x}$ from both intraseasonal and interannual scale. Besides, the governing equation for interannual SST includes the nonlinear zonal advection effect $-\mu(U T)$ and thermocline feedback $c_{1} \eta H$, which play important roles in triggering EP and $\mathrm{CP} \mathrm{El}$ Niño events, respectively (Jin and An 1999; An and Jin 2001; Jin et al. 2006). Below, we highlight the new physical mechanisms that are incorporated into the full model and play key roles in reproducing both the realistic ENSO diversity and the observed non-Gaussian statistical features.

First, we add a multiplicative noise $\sqrt{\left(2 d_{q} / k_{q}\right)\left(\bar{q}+q^{\prime}\right)^{+} \bar{q}^{+}} \dot{W}_{q}$ in the intraseasonal moisture $q^{\prime}$ equation, mimicking the irregular perturbation due to unresolved convective activity. This multiplicative noise has a similar form as that in convective activity equation $\sqrt{(2 \lambda / k)\left(\bar{a}+a^{\prime}\right) \bar{a}} \dot{W}_{a}$, which guarantee the nonnegative property of total moisture $q^{\prime}+\bar{q}$ and total convective activity $a^{\prime}+\bar{a}$. The value of interannual moisture $\bar{q}$ is obtained according to the conservation law of column-integrated moist static energy (MSE) $\theta_{e}$. It can be shown by a scaling argument that the leading-order balance in the MSE budget on the planetary/interannual scale is between moisture source $E_{q}=\alpha_{q} T$ and radiative heating/cooling effects that restore the actual MSE $\theta_{e}$ toward the background MSE $\bar{\theta}_{e}$, that is, $0=\alpha_{q} T-\left(1 / \tau_{\theta_{e}}\right)\left(\theta_{e}-\bar{\theta}_{e}\right)$. By taking the linearized form of MSE $\theta_{e}=\bar{q}+\bar{\theta}$, we can obtain the expression for interannual moisture,

$$
\bar{q}=\bar{\theta}_{e}+\tau_{\theta_{e}} \alpha_{q} T-\bar{\theta},
$$

where $\bar{\theta}_{e}$ is the background equivalent potential temperature (see Table 3 ), $\tau_{\theta_{e}}$ is the relaxation time scale, $\bar{\theta}$ is interannual potential temperature anomaly. It is worth mentioning that the nonnegative operator + is added in the multiplicative noise because these two quantities $\bar{q}+q^{\prime}$ and $\bar{q}$ become negative in some rare events during a long simulation.

Second, we add a nonlinear zonal advection term $-\mu(U T)_{x}$ with no ad hoc parameterization of the background SST gradient in the SST equation in the same manner as Chen and Majda (2016). In the presence of a mean easterly trade wind anomaly, Chen and Majda (2016) showed that this nonlinear 
TABLE 1. Governing equations of the full model and descriptions about the notation, variables, and coefficients.

\begin{tabular}{|c|c|c|c|}
\hline Time & Model & Governing equations & Description \\
\hline Intraseasonal & Atmosphere & $\begin{array}{c}\partial_{t} u^{\prime}-y v^{\prime}=\partial_{x} \theta^{\prime}-d_{u} u^{\prime} \\
y u^{\prime}=\partial_{y} \theta^{\prime} \\
\partial_{t} \theta^{\prime}-\left(\partial_{x} u^{\prime}+\partial_{y} v^{\prime}\right)=\bar{H} a^{\prime}-d_{\theta} \theta^{\prime} \\
\partial_{t} q^{\prime}+\bar{Q}\left(\partial_{x} u^{\prime}+\partial_{y} v^{\prime}\right)=-\bar{H} a^{\prime}-d_{q} q^{\prime}+\sqrt{\frac{2 d_{q}}{k_{q}}\left(\bar{q}+q^{\prime}\right)^{+} \bar{q}^{+}} \dot{W}_{q} \\
\partial_{t} a^{\prime}=\Gamma q^{\prime}\left(\bar{a}+a^{\prime}\right)-\lambda a^{\prime}+\sqrt{\frac{2 \lambda}{k}\left(\bar{a}+a^{\prime}\right) \bar{a}} \dot{W}_{a}\end{array}$ & $\begin{array}{l}\text { Intraseasonal atmospheric fields } \\
\quad u^{\prime}, v^{\prime}, \theta^{\prime}, q^{\prime}, a^{\prime} \\
\text { Shape parameters } k^{\prime}, k_{q}^{\prime} \\
\text { Notation } f^{+}= \begin{cases}f, & \text { if } f \geq 0 \\
0, & \text { else }\end{cases} \\
\text { Interannual moisture } \bar{q}=\bar{\theta}_{e}+\tau_{\theta_{e}} \alpha_{q} T-\bar{\theta}\end{array}$ \\
\hline Interannual & Atmosphere & $\begin{array}{c}-y \bar{v}=\partial_{x} \bar{\theta} \\
y \bar{u}=\partial_{y} \bar{\theta} \\
-\left(\partial_{x} \bar{u}+\partial_{y} \bar{v}\right)=\bar{H} \bar{a}-s^{\theta} \\
\bar{Q}\left(\partial_{x} \bar{u}+\partial_{y} \bar{v}\right)=-\bar{H} \bar{a}+s^{q}+E_{q}\end{array}$ & $\begin{array}{l}\text { Interannual atmospheric fields } \bar{u}, \bar{v}, \bar{\theta}, \bar{a} \\
\bar{H} \bar{a}=\frac{1}{1-\bar{Q}}\left(E_{q}+s^{q}-\bar{Q} s^{\theta}\right) \\
\text { Evaporation is parameterized as } \\
\quad E_{q}=\alpha_{q} T\end{array}$ \\
\hline & Ocean & $\begin{array}{c}\partial_{t} U-\varepsilon c_{1} Y V+\varepsilon c_{1} \partial_{x} H=\varepsilon c_{1} \tau_{x} \\
Y U+\partial_{Y} H=0 \\
\partial_{t} H+\varepsilon c_{1}\left(\partial_{x} U+\partial_{Y} V\right)=0\end{array}$ & $\begin{array}{l}\text { Interannual oceanic fields } U, V, H \\
\text { Wind stress is parameterized } \\
\quad \text { as } \tau_{x}=\gamma\left(\bar{u}+u^{\prime}\right)\end{array}$ \\
\hline & SST & $\partial_{t} T+\varepsilon \mu(U T)_{x}=-\varepsilon c_{1} \zeta E_{q}+\varepsilon c_{1} \eta H$ & $\begin{array}{l}\text { Parameter } \mu \text { measures the strength } \\
\text { of zonal advection }\end{array}$ \\
\hline Multidecadal & $\begin{array}{l}\text { Background } \\
\text { Walker } \\
\text { circulation }\end{array}$ & $d I_{\mathrm{wc}}=-\frac{1}{\tau_{\mathrm{wc}}}\left(I_{\mathrm{wc}}-I_{0}\right) d t+\sigma\left(I_{\mathrm{wc}}\right) d W_{t}$ & $\begin{array}{l}\text { External sources of moistening } \\
\qquad s^{q}=s^{\theta}+I_{\mathrm{wc}} A_{0} \cos \left(\frac{2 \pi}{L} x\right) \\
\text { The equilibrium PDF is shown in Fig. 1a }\end{array}$ \\
\hline
\end{tabular}

zonal advection plays a key role in recovering the CP El Niño events with realistic amplitude and duration.

Third, we introduce a simple stochastic equation to govern the multidecadal variation of the Walker circulation. The motivation of coupling the background Walker circulation into the full model is twofold: 1) inducing the background trade wind to trigger CP El Niño events (Chen and Majda 2016) and 2) resembling the modulation of ENSO dynamics by the multidecadal variation of the Walker circulation in the observation (Knutson et al. 1997; Dong et al. 2006). Here we use a nondimensional variable $I_{\mathrm{wc}}$ to represent the difference between external sources of cooling and moistening $s^{\theta}$ and $s^{q}$,

$$
s^{q}=s^{\theta}+I_{\mathrm{wc}} A_{0} \cos \left(\frac{2 \pi}{L} x\right),
$$

where the constant $A_{0}$ is chosen to normalize the value of $I_{\mathrm{wc}}$ and the zonal profile $\cos [(2 \pi / L) x]$ is chosen for simplification. This variable $I_{\mathrm{wc}}$ is governed by a simple stochastic equation,

$$
d I_{\mathrm{wc}}=-\frac{1}{\tau_{\mathrm{wc}}}\left(I_{\mathrm{wc}}-I_{0}\right) d t+\sigma\left(I_{\mathrm{wc}}\right) d W_{t},
$$

where $\tau_{\mathrm{wc}}=10$ years is a multidecadal relaxation time scale, and the mean value $I_{0}=0.34$ corresponds to the background heating that peaks to the western end of the Pacific. The term $d W_{t}$ stands for white noise perturbations during a time step $d t$. The recent observation evaluated the multidecadal trends of the Walker circulation in a bounded strength (Meng et al. 2012; L'Heureux et al. 2013). To automatically restrict the value of $I_{\mathrm{wc}}$ between -1.0 and 1.0, we consider a multiplicative noise coefficient (see Fig. 1a) based on the stochastic theory (Averina and Artemiev 1988; Chen and Majda 2020). As shown by Table 2, the variable $I_{\mathrm{wc}}$ switches the solution toward the EP (CP) El Niño regime without (with) the background Walker circulation when its value is close to $0(1.0)$. There is also a certain probability that the value of $I_{\mathrm{wc}}$ goes below 0 (see Fig. 1a), leading to the quiescent state with a slightly negative but nearly zero SST throughout the ocean domain. Recall that in Eq. (1), the magnitude of intraseasonal state-dependent noise is modulated by SST in a way that warmer (colder) SST anomalies lead to a stronger (weaker) noise for wind bursts. The cold SST anomalies associated to a weakening background Walker circulation provide unfavorable conditions for triggering wind bursts and thus suppress ENSO events. Besides, the nonlinear zonal advection of SST is switched on in a fixed strength, while the background equivalent potential temperature $\bar{\theta}_{e}$ is allowed to vary depending on the value of $I_{\mathrm{wc}}$. To trigger more EP El Niño events, we choose to let the magnitude of $\bar{\theta}_{e}$ increase gradually to become 2.5 times stronger when the value 
(a)

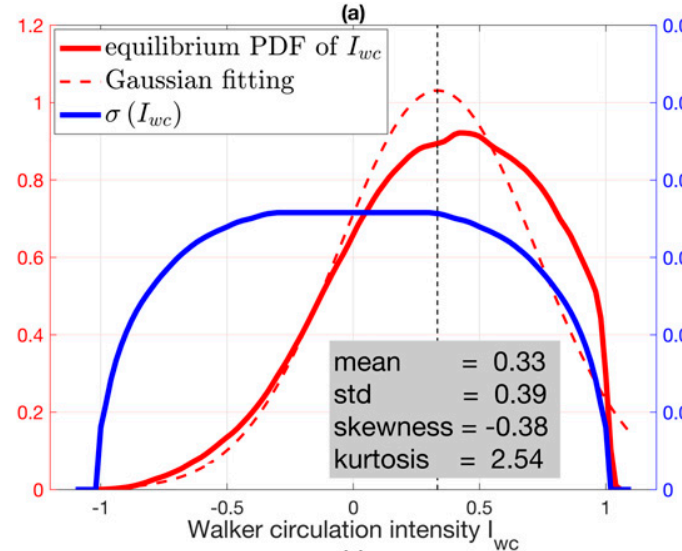

(c)

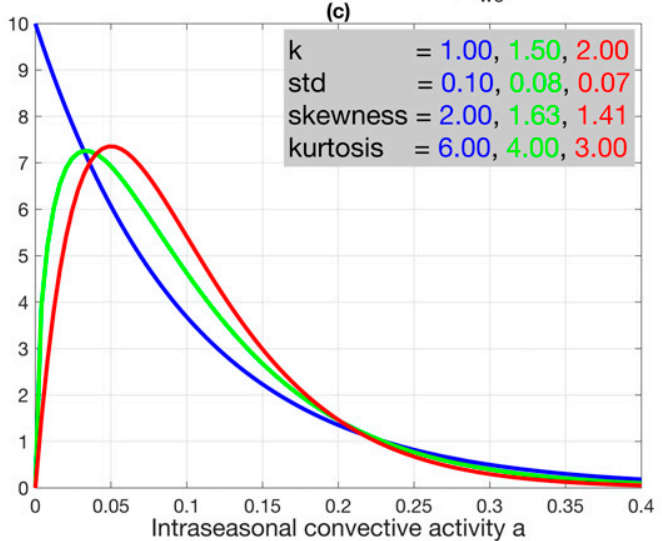

(b)
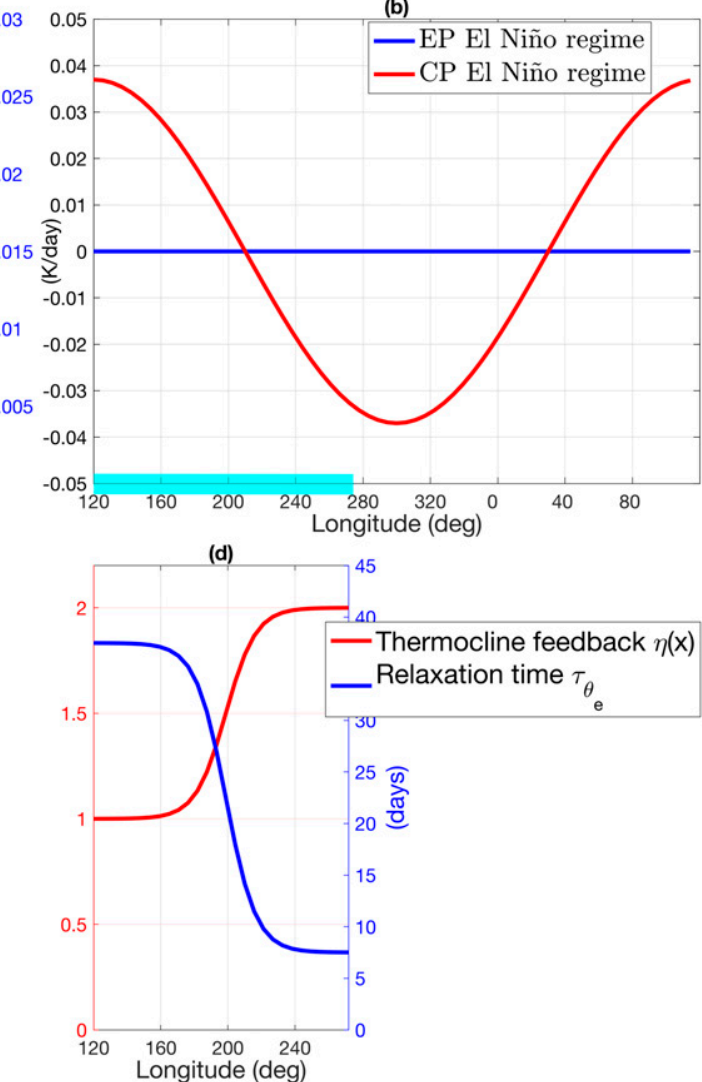

FIG. 1. (a) The equilibrium PDF of Walker circulation intensity $I_{\mathrm{wc}}$ and its Gaussian distribution fitting in the left $y$ axis and the multiplicative noise coefficient profile $\sigma\left(I_{\mathrm{wc}}\right)$ in the right $y$ axis. The first four statistical moments are included in the gray box. (b) The zonal profiles of external heating $\bar{H} \bar{a}-s^{\theta}$ in the EP and CP El Niño regimes, respectively. The bold light blue line indicates the longitude range of the ocean domain. (c) The gamma distribution for intraseasonal convective activity $a$ with different values of shape parameter $k$. Their second, third, and fourth statistical moments are included in the gray box. (d) The zonal profiles of the thermocline feedback parameter $\eta(x)$ in the left $y$ axis and the relaxation time scale of equivalent potential temperature $\tau_{\theta_{e}}$ in the right $y$ axis over the ocean domain.

of $I_{\mathrm{wc}}$ gets closer to 0 in the $\mathrm{EP} \mathrm{El}$ Niño regime. It is worthwhile to note that Eq. (3) is an independent governing equation for the multidecadal variation of the background Walker circulation, so it does not feel the feedback from intraseasonal and interannual fields. If $I_{\mathrm{wc}} \neq 0$, the imbalance of external sources between moistening $s^{q}$ and cooling $s^{\theta}$ will drive both the interannual atmosphere and ocean dynamics (see the second row in Table 1) as the background state. This background state varies on the multidecadal time scale as the variable index $I_{\mathrm{wc}}$ does. In the presence of the multidecadal Walker circulation, the interannual variables such as ocean current $U$ and SST $T$ contain not only the interannual component from the ENSO dynamics but also the multidecadal component from the background Walker circulation. Therefore, the term $-\mu(U T)_{x}$ includes both the linear and nonlinear zonal advection effects of SST.

Table 3 summarizes the parameters and constants in the full model, most of which are set in the same value as Thual et al. (2018). The values of the remaining parameters are chosen

TABLE 2. The multidecadal variation of background Walker circulation $I_{\mathrm{wc}}$ modulates the switching among the quiescent, EP El Niño, and CP El Niño regimes.

\begin{tabular}{lccc}
\hline \hline \multicolumn{1}{c}{ Value } & $I_{\mathrm{wc}} \sim-1$ & $I_{\mathrm{wc}} \sim 0$ & $I_{\mathrm{wc}} \sim 1$ \\
\hline Regime & Quiescent & EP El Niño & CP El Niño \\
Background Walker circulation & Yes (cold SST) & No & Yes (warm SST) \\
Nonlinear SST advection & $\mu=0.04$ & $\mu=0.04$ & $\mu=0.04$ \\
State-dependent wind noise & Weak & Strong & Moderate \\
Background equivalent potential temperature in modulating wind bursts & $2.5 \bar{\theta}_{e}$ & $2.5 \bar{\theta}_{e}$ & $\bar{\theta}_{e}$ \\
\hline
\end{tabular}


TABLE 3. The values and descriptions of all model parameters. The number in parentheses is its nondimensional value after the scaling.

\begin{tabular}{lcl}
\hline \hline Parameter & Value & \multicolumn{1}{c}{ Description } \\
\hline$C$ & 0.05 & Ratio of ocean and atmosphere phase speed \\
$\varepsilon$ & 0.1 & Froude number \\
$c_{1}=c / \varepsilon$ & 0.5 & Ratio between $c$ and $\varepsilon$ \\
$\bar{H}$ & $10 \mathrm{~K} \mathrm{day}^{-1}(22)$ & Convective heating rate factor \\
$\bar{Q}$ & 0.9 & Mean vertical moisture gradient \\
$\Gamma$ & $0.2 \mathrm{~K} \mathrm{day}^{-1}(1.66)$ & Convective growth/decay rate \\
$d_{u}, d_{\theta}, d_{q}, \lambda$ & $1 / 30 \mathrm{day}^{-1}(0.11)$ & Atmosphere dissipation \\
$k_{q}, k$ & 2.0 & Shape parameter in $q^{\prime}, a^{\prime}$ multiplicative noise \\
$\gamma$ & 6.53 & Wind stress coefficient \\
$\mu$ & 0.04 & Nonlinear zonal advection coefficient \\
$\zeta$ & 8.7 & Latent heating exchange coefficient \\
$\alpha_{q}$ & 0.2 & Latent heating factor \\
$r_{W}$ & 0.5 & Western boundary reflection coefficient in ocean \\
$r_{E}$ & 1.0 & Eastern boundary reflection coefficient in ocean \\
$I_{0}$ & 0.34 & Mean value of $I_{\mathrm{wc}}$ \\
$\tau_{\mathrm{wc}}$ & 10 years $\left(1.1 \times 10^{3}\right)$ & Relaxation time scale of $I_{\mathrm{wc}}$ \\
$A_{0}$ & Maximum strength of the excess of external moistening source $s^{q}$ \\
$\bar{\theta}_{e}$ & $0.375[1+0.6 \times \cos (2 \pi x / L)] \mathrm{K}$ & Background equivalent potential temperature \\
\hline
\end{tabular}

under the guidance of the observation. In particular, the shape parameters $k_{q}$ and $k$ play a central role in determining the nonGaussian features of intraseasonal flow fields (see discussion in section 3). The magnitude coefficient $\mu$ is chosen to have an intermediate value 0.04 so that the nonlinear zonal advection $c_{1} \zeta E_{q}$ has a comparable contribution as the thermocline feedback $c_{1} \eta H$ in the SST budget. The mean value of $I_{\mathrm{wc}}$ is fixed at 0.34 so that the occurrence frequency of EP and CP El Niño events matches the observation. Notably, sensitivity tests show that key features of ENSO events in the simulation are unchanged when varying these parameters, reflecting the model robustness.

Before the numerical implementation, we first project the meridional profiles of all physical variables onto the first and third parabolic cylinder functions, the leading two symmetric modes in tropical wave dynamics (Majda 2003). The model has a $40000-\mathrm{km}$ atmospheric domain for the tropical belt and a $17500-\mathrm{km}$ ocean domain sitting to its west. The western end of the ocean domain is set at the longitude $120^{\circ} \mathrm{E}$. We then solve the full model numerically by the method of lines, including the spectral method for spatial discretization $(625-\mathrm{km}$ grid spacing) and the Euler scheme for time stepping $(0.8 \mathrm{~h}$ each time step). The nonlinear zonal advection of SST is solved by the upwind finite volume method to guarantee the conservation law (LeVeque et al. 2002; Bouche et al. 2011). All stochastic equations with the multiplicative noise are solved by the EulerMaruyama method. Periodic boundary conditions are imposed in the atmospheric models while reflecting boundary conditions are imposed in the ocean model (reflection coefficients are listed in Table 3). The simulation in section 4 runs for 2000 years with the last 1800-yr solutions for diagnostic analysis.

b. External sources of moistening and cooling, thermocline feedback coefficient, relaxation time scale, and gamma distribution

Besides parameters and constants, there are still several external forcing and coefficient profiles that need to be determined before we solve the full model numerically. Besides, we also summarize some general statistical features of gamma distribution, which should be useful for our physical interpretation in section 3 .

Figure $1 \mathrm{~b}$ shows the zonal profiles of total heating $\bar{H} \bar{a}-s^{\theta}$ due to the external source of moistening $s^{q}$ and cooling $s^{\theta}$ in the $\mathrm{EP}$ and CP El Niño regimes. In the CP El Niño regime $\left(I_{\mathrm{wc}} \sim\right.$ 1 ), the excess of moistening forcing $s^{q}$ induces total heating that peaks to the west of the ocean domain, driving a planetaryscale wind convergence to the west with prevailing easterly winds over the most ocean domain. In the EP El Niño regime $\left(I_{\mathrm{wc}} \sim 0\right)$, the total heating vanishes due to the exact balance between $s^{q}$ and $s^{\theta}$. Figure $1 c$ shows the equilibrium PDF and the statistical moments of gamma distribution with different values of shape parameter $k$. In detail, the gamma distribution with $k=2.0$ has the kurtosis equal to 3 , the same as the Gaussian distribution, but a positive skewness (1.41), reflecting the asymmetric property of the PDF itself. As the value of $k$ decreases from 2.0 to 1.0 , the kurtosis increases dramatically from 3.0 to 6.0, indicating a fat-tailed PDF and the increasing likelihood of the occurrence of extreme events. Meanwhile, both the standard deviation and skewness increase. Figure 1d shows the thermocline feedback coefficient $\eta(x)$ and the relation time scale $\tau_{\theta_{e}}$ for restoring actual MSE $\theta_{e}$ toward its background profile. The magnitude of $\eta(x)$ is double to the east compared to the west, consistent with the fact that the thermocline feedback mechanism for the ENSO is more efficient over the EP due to the shoaling thermocline there. As for the MSE relaxation time scale $\tau_{\theta_{e}}$, its value to the west is chosen to be 5 times as large as that to the east, mimicking the significant MSE variability and its long relaxation time scale over the western Pacific (WP) in the observation.

\section{c. SST observational data}

In this study, two observational SST data are utilized to compare with the model output in terms of the equilibrium 
PDF and frequency spectrum of ENSO events as well as individual case studies. The first SST data are from the OISST reanalysis product (Reynolds et al. 2007; https://www.ncdc. noaa.gov/oisst). Here these daily SST data are averaged meridionally within $5^{\circ} \mathrm{N}-5^{\circ} \mathrm{S}$ in the tropical Pacific $\left(120^{\circ} \mathrm{E}-80^{\circ} \mathrm{W}\right)$ and cover the period from January 1982 to September 2016. In particular, we focus on three Niño indexes that are calculated by taking the average of SST anomalies about different ocean regions, including 1$)$ the Niño-4 region $\left.\left(160^{\circ} \mathrm{E}-150^{\circ} \mathrm{W}\right), 2\right)$ the Niño-3.4 region $\left(170^{\circ}-120^{\circ} \mathrm{W}\right)$, and 3$)$ the Niño-3 region $\left(150^{\circ}-\right.$ $90^{\circ} \mathrm{W}$ ). After removing the climatology (35-yr mean), we also apply a 90-day-centered running mean on the data so that the resulting SST anomalies should mostly represent its interannual variability. The second SST data are from the Met Office Hadley Centre Sea Ice and Sea Surface Temperature dataset (HadISST; Rayner et al. 2003) and cover a 150-yr period from 1870 to 2019. To obtain the Niño-4, Niño-3.4, and Niño-3 indexes, we first take averaging of this monthly SST data over the three Niño regions. Then the resulting time series of SST is further detrended with its climatological seasonal cycle removed.

\section{Irregular and non-Gaussian features of wind bursts and the MJO}

The full model in Table 1 describes a multiple-time-scale interaction in a way that the intraseasonal atmosphere contributes to the triggering of ENSO events through wind bursts over the ocean and also feels the modulation effect from interannual SST and multidecadal variability of the background Walker circulation. Here we focus on the intraseasonal dynamics in the warm pool scenario with fixed background fields $\left(s^{\theta}, s^{q}, \bar{\theta}_{e}\right)$ but no interannual and multidecadal variation. The goal is to investigate how well the MJO and wind bursts are simulated in this stochastic intraseasonal model in terms of their irregular patterns and nonGaussian features. Without loss of generality, we only consider a specific case with both shape parameters of the multiplicative noise $k_{q}=k=2.0$. This simulation runs for 20 years with the last 18-yr solution for diagnostic analysis.

Figures 2a-c shows the 150-day Hovmöller diagrams for intraseasonal fields including heating $\bar{H} a^{\prime}$, zonal winds $u^{\prime}$, and moisture anomaly $q^{\prime}$. All these three fields reach their maximum strength near the warm pool center and exhibit irregular patterns due to the multiplicative noise. In Fig. 2a, heating anomalies prevail over the warm pool in a scattered pattern, emanating many eastward- and westward-propagating waves on both sides. Similarly, moisture anomalies in Fig. 2c exhibit an irregular pattern and small-scale features with both standing and propagating wave modes near the warm pool. In contrast, zonal winds in Fig. $2 \mathrm{~b}$ feature a large-scale and regular pattern when compared with heating and moisture anomalies. A few planetary-scale envelopes of wind bursts are first initialized to the west of the warm pool, then go across the warm pool center and finally enter the ocean domain, consistent with the realistic propagation of the MJO in the observation (Zhang 2005). Meanwhile, many small-scale westward-moving waves are embedded in the eastward-moving planetary-scale envelops, resembling the observed multiscale features of the MJO (Nakazawa 1988; Chen et al. 1996). Notably, these large-scale wind bursts could last longer than 10 days, presumably due to the nonlinear interplay between moisture and convective activity as well as the multiplicative noise.

Figures $2 \mathrm{~d}-\mathrm{f}$ shows the wavenumber-frequency diagrams for these three intraseasonal fields, which look similar to the previous study of Thual et al. (2014). In detail, both heating and zonal wind fields feature three dominant wave spectrum bands, including the eastward-moving MJO in 30-90 days, the westwardmoving moist Rossby waves, and the domainwide oscillations in fewer than 20 days. As for zonal winds, its eastward-moving wave spectra is dominated by the MJO mode, a major source of eastward-moving wind bursts over the WP (Seiki and Takayabu 2007a,b). Besides, the westward-moving wave spectra of wind bursts are contributed by both slow-moving moist Rossby waves and fast-moving dry Rossby waves in wavenumber $1-3$. It is worth noting that due to the simplicity of the model, the spectral peaks of the simulated MJO extend from wavenumber 2 to 10, in contrast to the observation (Puy et al. 2016).

Figures $2 \mathrm{~g}-\mathrm{i}$ show the equilibrium PDF of these three intraseasonal fields. For heating $\bar{H} a^{\prime}$, its equilibrium PDF in Fig. 2 g looks similar to the gamma distribution in Fig. $1 \mathrm{c}$ with a one-sided fat tail, representing the high occurrence of strong events. Although the shape parameter $k$ is set to be 2.0, its skewness and kurtosis values $(1.61,6.37)$ are both larger than those of gamma distribution $(1.41,3.0)$, resulting from the nonlinear term $\Gamma q^{\prime}\left(\bar{a}+a^{\prime}\right)$ in convective activity equation and the multiplicative moisture noise. These non-Gaussian features of heating are akin to the high occurrence of strong magnitude events such as extreme precipitation (Jones et al. 2004). Since this heating directly drives wind fields, the equilibrium PDF of $u^{\prime}$ also has a kurtosis value (3.18) that is slightly larger than 3.0, indicating the occurrence of strong wind bursts. Meanwhile, the skewness of $u^{\prime}, q^{\prime}$ fields are both larger than zero, reflecting significant asymmetry in intraseasonal zonal wind and moisture fields.

Besides this specific case, we also undertake a few sensitivity tests (not shown) by varying the values of these two shape parameters $k_{q}$ and $k$. In general, varying $k_{q}, k$ does not qualitatively change the spatiotemporal patterns and statistical features of these three fields, but only their absolute magnitude. After reducing $k_{q}$ and $k$ from 2.0 to 1.0, the wave spectra of the MJO mode in all intraseasonal fields become wider and stronger, consistent with the theoretical results of the gamma distribution in Fig. 1c. Such an intensification effect stems from the nonlinear interplay between $q^{\prime}$ and $a^{\prime}$ as well as the multiplicative noise in both fields. As for the statistical features, the skewness and kurtosis of these three intraseasonal fields all increase after reducing the value of shape parameters $k_{q}$ and $k$. Moreover, reducing $k_{q}$ leads to a larger value of the skewness and kurtosis of the equilibrium PDF, reflecting the higher efficiency of the multiplicative moisture noise in triggering extreme intraseasonal fields.

\section{ENSO diversity and non-Gaussian features in the full model}

As shown by Table 2, the value of $I_{\mathrm{wc}}$ controls the strength of background trade winds and intraseasonal wind bursts and thus determines the switching between EP and CP El Niño regimes. 

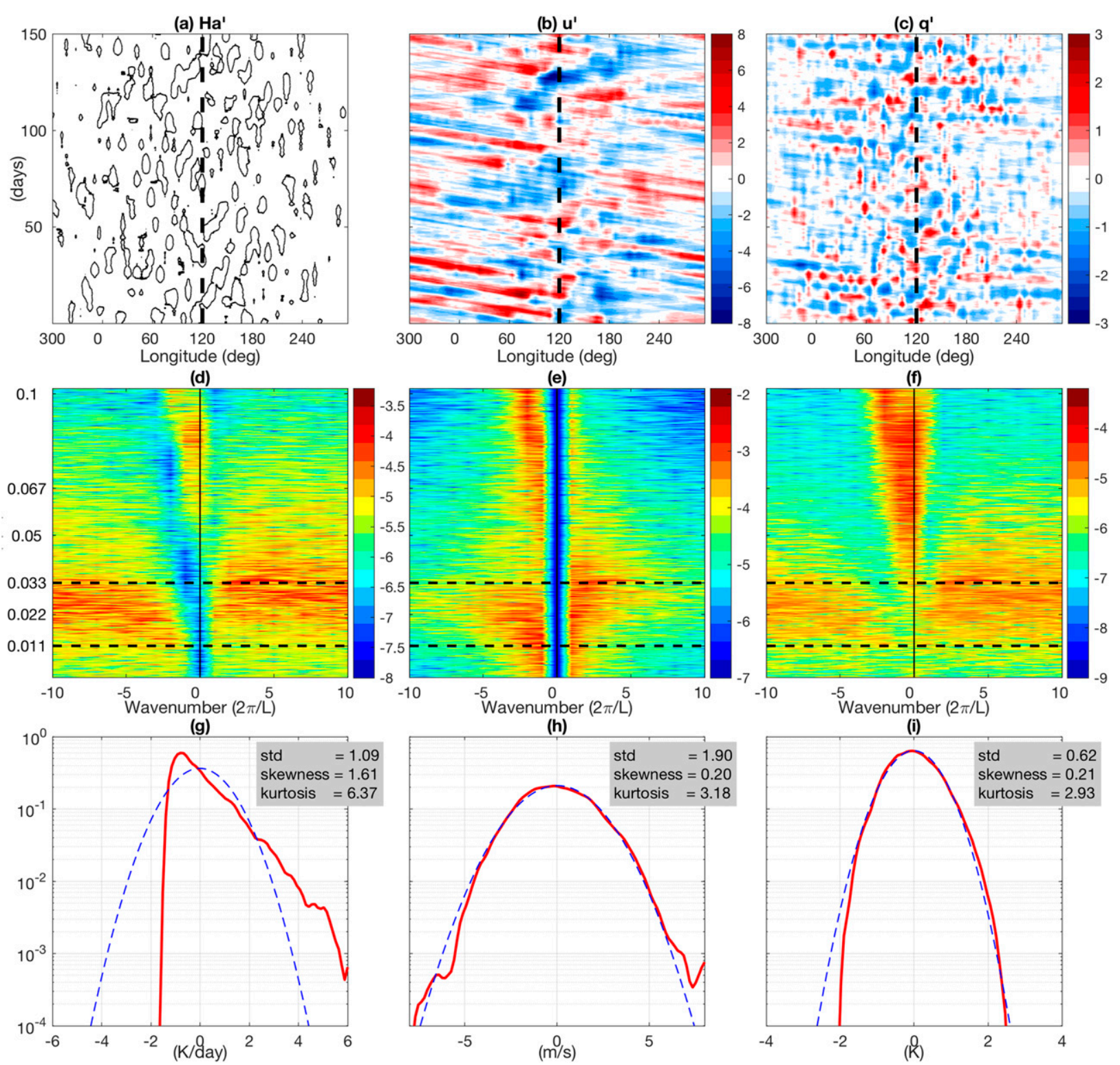

FIG. 2. Spatiotemporal variability of intraseasonal flow fields [heating $\bar{H} a^{\prime}\left(\mathrm{K}\right.$ day $\left.^{-1}\right)$, zonal winds $u^{\prime}\left(\mathrm{m} \mathrm{s}^{-1}\right)$, and moisture anomaly $q^{\prime}$ $(\mathrm{K})$; units are dimensional] in the case with shape parameters $k=2.0$ and $k_{q}=2.0$. (a)-(c) Their Hovmöller diagrams during a 150-day period. The dashed line indicates the warm pool center and also the western end of the ocean domain. (d)-(f) Their log10-scale wavenumber-frequency spectra based on the last 18-yr model output. The two dashed lines indicate the period of 30 and 90 days, respectively. (g)-(i) Their log-scale equilibrium PDF based on 18-yr time series at the longitude $X=5000 \mathrm{~km}$ over the western ocean domain. The secnd, third, and fourth statistical moments are summarized in the gray box. The blue dashed curves are Gaussian fits that are parabola in the log-scale plotting.

To first investigate each regime separately, we fix $I_{\mathrm{wc}}$ at the extreme value so that only EP or CP Niño events arise in each simulation. Specifically, we focus on the EP El Niño scenario in subsection $4 \mathrm{a}$ by fixing the value of $I_{\mathrm{wc}}$ at 0 . Then we consider the CP El Niño scenario in subsection $4 b$ by fixing the value of $I_{\mathrm{wc}}$ at 1.0. In subsection $4 \mathrm{c}$, the variable $I_{\mathrm{wc}}$ varies between -1.0 and 1.0 as governed by the simple stochastic equation in Eq. (3). The goal of this section is to investigate the skill of the full model in capturing the ENSO diversity and the non-
Gaussian features of SST in comparison with the observation. (For better visualization, all interannual fields shown in Figs. 4-6 also contain the background state driven by the multidecadal variation of the Walker circulation.)

\section{a. EP El Niño scenario}

The EP El Niño scenario includes no background Walker circulation if the value of $I_{\mathrm{wc}}$ is close to 0 . This is consistent with the observation that easterly trade winds over the Pacific, one 

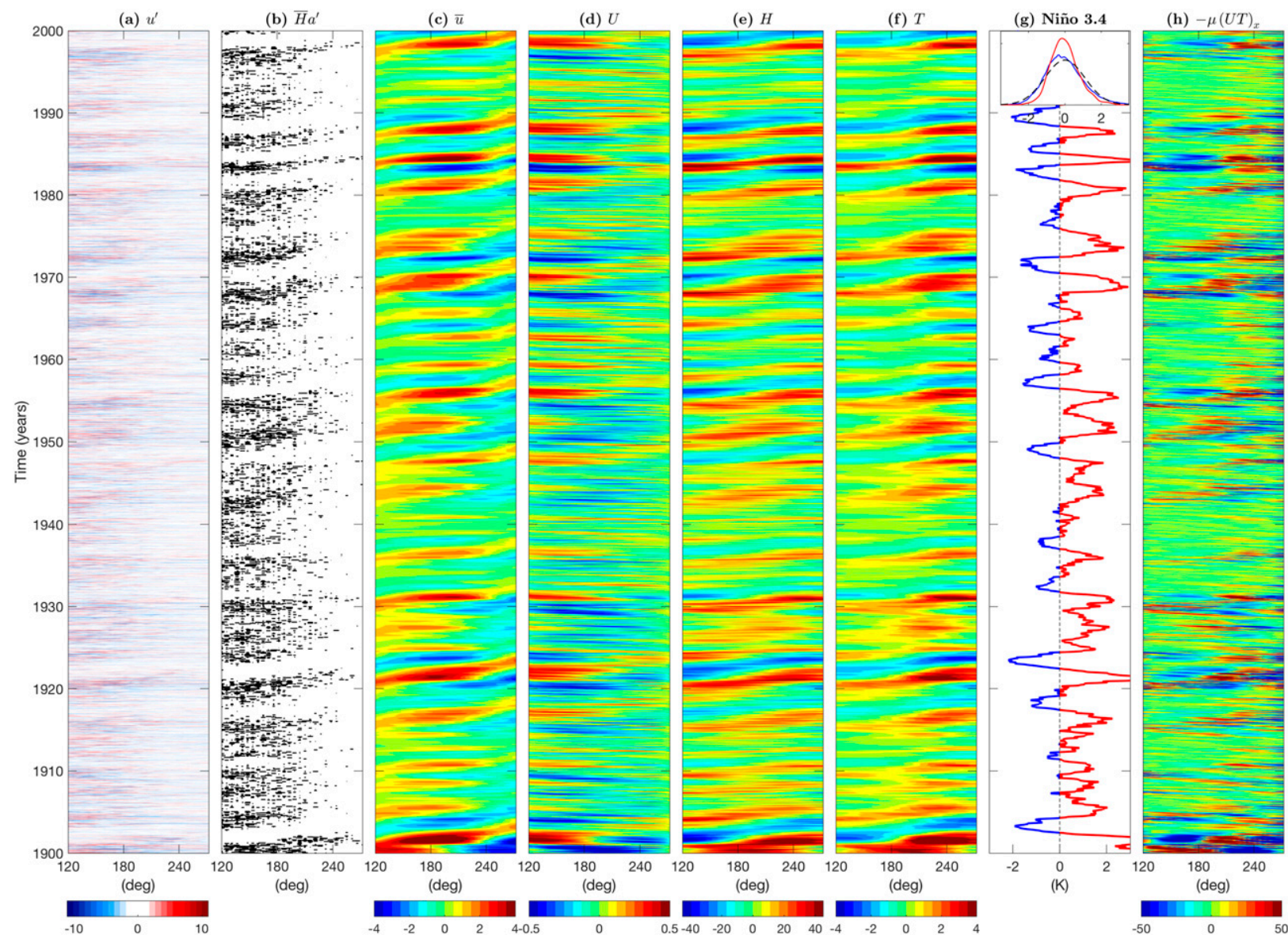

FIG. 3. Hovmöller diagrams for (a) intraseasonal zonal winds $u^{\prime}\left(\mathrm{m} \mathrm{s}^{-1}\right)$, (b) intraseasonal convective activity $\bar{H} a^{\prime}\left(\mathrm{K}\right.$ day $\left.{ }^{-1}\right)$, (c) interannual zonal winds $\bar{u}\left(\mathrm{~m} \mathrm{~s}^{-1}\right)$, (d) ocean current $U\left(\mathrm{~m} \mathrm{~s}^{-1}\right)$, (e) thermocline depth $H(\mathrm{~m})$, (f) SST $T(\mathrm{~K})$, (g) the time series of SST in the Niño-3.4 region $(\mathrm{K})$, and $(\mathrm{h})$ nonlinear zonal advection of equatorial SST term $-\mu(U T)_{x}\left(\mathrm{~K} \mathrm{yr}^{-1}\right)$ from the last 100 -yr simulation in the EP El Niño scenario. The black shading in (b) indicates $\bar{H} a^{\prime}$ anomalies that are larger than $4 \mathrm{~K}$. In $(\mathrm{g})$, the blue curve inside the top box shows the equilibrium PDF of SST in the Niño-3.4 region and the dashed curve is its Gaussian distribution fitting. The standard deviation, skewness and kurtosis of this equilibrium PDF are 1.09, 0.59, and 4.10. The PDF of the Niño-3.4 index based on the HadISST dataset is shown in red for comparison. Units are dimensional.

branch of the Walker circulation, are typically weak during the EP El Niño episodes (Clarke 2008). Meanwhile, the equivalent potential temperature is assumed to be strong over the WP, providing favorable convective conditions for triggering wind bursts [see Eq. (1)].

Figure 3 shows the Hovmöller diagrams for multiple intraseasonal and interannual fields from the last $100-\mathrm{yr}$ simulation in the EP El Niño scenario. As indicated by Fig. 3f, the model reproduces both the moderate $\mathrm{El}$ Niño (e.g., the one at year 1935) and super El Niño (e.g., the one at year 1920) as well as La Niña events. These EP El Niño events are manifested by warm SST anomalies that are first initialized over the WP, move eastward across the date line, intensify, and then decay over the EP, followed by La Niña events with eastward-moving cold SST anomalies in a weak magnitude. It is worth mentioning that almost all the model-simulated EP El Niño events are initialized over the WP, in contrast to the observation (Timmermann et al. 2018). This deficiency is presumably due to the prescribed nonnegligible thermocline feedback over the WP (see Fig. 1d) and the lack of counteracting damping processes there in this simplified model. Intense wind bursts $u^{\prime}$ in Fig. 3a and heating anomalies $\bar{H} a^{\prime}$ in Fig. $3 b$ occur over the regions with warm SST anomalies in Fig. 3f. Meanwhile, these two intraseasonal fields are mostly confined over the WP, resembling the prevalent convection and wind bursts there in the observation (Seiki and Takayabu 2007a,b). For the interannual atmosphere in Fig. 3c, these eastward-moving warm SST anomalies are accompanied by westerlies (easterlies) to the west (east), reflecting the presence of heating driven wind convergence in the lower troposphere. In the interannual ocean, most significant eastward and westward ocean currents in Fig. 3d occur over the WP, while high-frequency waves that move on both directions are found all over the ocean domain. Besides, the thermocline depth anomalies in Fig. 3e feature a similar spatiotemporal pattern as SST in Fig. 3f, consistent with the dominant role of the thermocline feedback for El Niño 


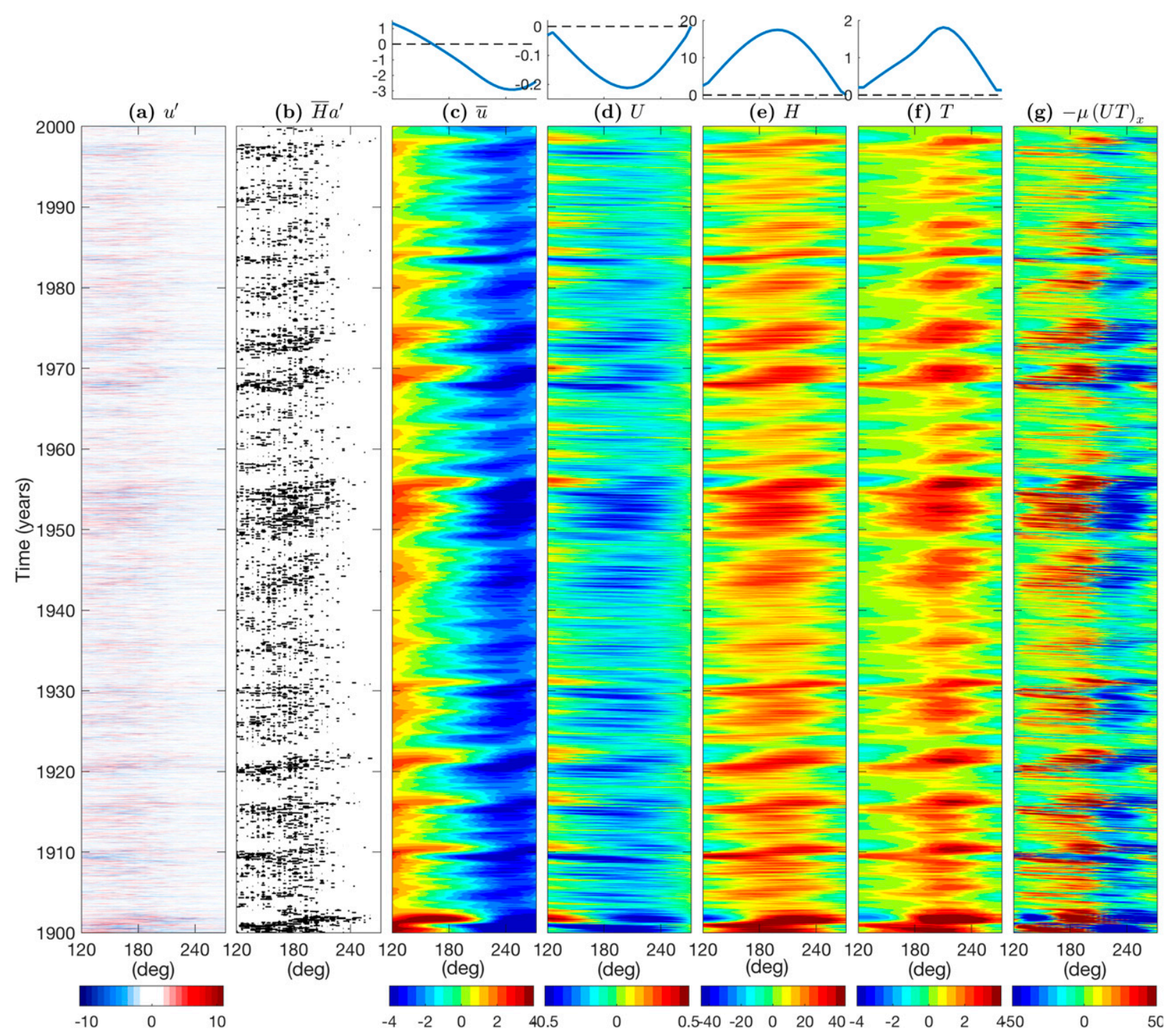

FIG. 4. Hovmöller diagrams for (a) intraseasonal zonal winds $u^{\prime}\left(\mathrm{m} \mathrm{s}^{-1}\right)$ (b) intraseasonal convective activity $\bar{H} a^{\prime}\left(\mathrm{K}\right.$ day $\left.{ }^{-1}\right)$, (c) interannual zonal winds $\bar{u}\left(\mathrm{~m} \mathrm{~s}^{-1}\right)$, (d) ocean current $U\left(\mathrm{~m} \mathrm{~s}^{-1}\right)$, (e) thermocline depth $H(\mathrm{~m})$, (f) SST $T(\mathrm{~K})$, and (g) nonlinear zonal advection of equatorial SST term $-\mu(U T)_{x}\left(\mathrm{~K} \mathrm{yr}^{-1}\right)$ from the last 100-yr simulation in the CP El Niño scenario. The black shading in (b) indicates $\bar{H} a^{\prime}$ anomalies that are larger than $4 \mathrm{~K}$. The curves above (c)-(f) show the climatological mean of corresponding fields below. Units are dimensional.

events (Jin and An 1999). Figure 3g shows the time series of Niño-3.4 index with realistic irregularity and amplitude asymmetry between El Niño and La Niña phases. The noticeable deviation from the Gaussian distribution indicates the significant non-Gaussian features of SST, including nonzero skewness due to ENSO amplitude asymmetry and large kurtosis due to super El Niño events. These non-Gaussian features are also significant in the PDF of the observed Niño-3.4 index based on the HadISST data. Figure $3 \mathrm{~h}$ shows the evolution of the nonlinear zonal advection term, which exhibits significant eventto-event differences. Such a zonal advection effect that lacks coherent structures differs from that in the CP El Niño scenario (see Fig. 4g), reflecting the fact that the zonal advection effect does not play an essential role in the EP Niño mechanisms.
It is worthwhile mentioning that this EP model (as well as the full model to be described below) retains the fundamental mechanisms revealed by previous studies. For example, Thual et al. (2016) highlighted the direct causal effect of wind bursts on ENSO variability, which is also evident here in Figs. 3a and 3f. Chen et al. (2018) emphasized the preferred wind structure with easterly wind bursts first building up heat content and WWBs triggering the El Niño. A similar ocean current structure with westward current leading eastward current over the WP is also seen in Fig. 3d. Comparison between simulations with/without the multiplicative noise (not shown) indicates that the long-lasting wind bursts resulting from the multiplicative noise are more efficient to trigger the ENSO. Also, the El Niño between years 1926 and 1930 of the simulation is akin 
to the delayed super El Niño in 2014-16 (Levine and McPhaden 2016), presumably due to the long-lasting easterly/westerly wind bursts. Notably, the nonlinear zonal advection of SST only plays a secondary role in inducing the EP Niño, in contrast to its key role in the CP El Niño scenario (see Fig. 4g).

\section{b. CP El Niño scenario}

In contrast to the EP El Niño scenario, the CP El Niño scenario feels the presence of the Walker circulation and the nonlinear zonal advection of SST if the value of $I_{\mathrm{wc}}$ is near 1.0. Chen and Majda (2016) first pointed out that a mean easterly trade wind anomaly and the nonlinear zonal advection with no ad hoc parameterization of the background SST gradient are two necessary conditions for triggering the CP El Niño events.

Figure 4 shows the Hovmöller diagrams for multiple intraseasonal and interannual fields from the last 100-yr simulation in the CP El Niño scenario. As shown in Fig. 4f, the eastwardmoving SST anomalies reach their maximum strength over the $\mathrm{CP}$, farther west than the EP El Niño in Fig. 3f. Moreover, these El Niños typically occur in a series of moderate magnitude events, resembling the observed series of 5-yr CP El Niño during the 1990s (Freund et al. 2019). Similar to Figs. 3a and 3b, the wind bursts in Fig. 4a and heating anomalies in Fig. 4b are confined over the WP, whose magnitudes get intensified (suppressed) when the SST is warm (cold). Unlike Figs. 3c and $3 \mathrm{~d}$, the wind bursts in Fig. $4 \mathrm{c}$ and ocean currents in Fig. 4d move eastward in a much shorter distance. Besides, the thermocline depth anomalies in Fig. 4e exhibit a similar spatiotemporal pattern as the SST in Fig. 4f.

As shown by the top panels above Figs. 4c-f, the background Walker circulation features a low-tropospheric wind convergence, the domainwide westward currents, deep thermocline depth anomalies, and warm SST anomalies with their maximum strength reached over the CP. In the presence of zonal advection of SST, this background westward current tends to transport warm water westward over the EP, while the anomalous eastward current in Fig. 4d leads the eastward displacement of the warm pool edge. This is consistent with the SST budget analysis in Fig. $4 \mathrm{~g}$ that the nonlinear zonal advection term induces warming over the western and central Pacific but cooling over the EP. Notably, this budget analysis highlights the important contribution of both background and anomalous fields in the zonal advection mechanisms, contrasting significantly with previous studies that assume a linear advection and require ad hoc parameterization of background SST gradient (Jin and An 1999; Dewitte et al. 2013; Ren and Jin 2013). In general, the nonlinear zonal advection term includes not only the advection of mean SST by anomalous zonal current, but also the advection of anomalous SST by anomalous zonal current. The former has been shown to play a crucial role in the CP Niño through SST budget analysis (Kug et al. 2009), while the latter was recently proposed by Chen and Majda (2016) as a complementary important mechanism.

\section{c. ENSO diversity in the presence of dynamic Walker circulation}

The full model allows the variable $I_{\mathrm{wc}}$ to vary as governed by the simple stochastic equation in Eq. (3), which describes the multidecadal variation of the background Walker circulation. Thus the simulation covers all possible regimes, including the CP El Niño regime $\left(I_{\mathrm{wc}} \sim 1.0\right)$, the EP El Niño regime $\left(I_{\mathrm{wc}} \sim 0\right)$, and the quiescent state $\left(I_{\mathrm{wc}} \ll 0\right)$.

Figure 5 shows the Hovmöller diagrams for equatorial SST from the last $800-\mathrm{yr}$ simulation of the full model. Due to its stochastic nature, the full model reproduces great ENSO diversity over this long period, including the quasi-regular moderate traditional El Niño, the super El Niño, and the CP El Niño as well as the La Niña with many realistic features. In detail, the EP El Niño (e.g., the one between year 1394 and 1397) is typically characterized by eastward-moving warm SST anomalies that are first initialized over the WP in a weak magnitude, then go across the $\mathrm{CP}$ with an intensifying magnitude, and eventually reach the EP in the maximum strength. This intensification of warm SST anomalies over the EP is related to the relatively strong thermocline feedback there (see Fig. 1d). The EP El Niño is typically followed by a La Niña. Unlike the EP El Niño, the CP El Niño (e.g., the one near year $1410)$ is typically characterized by warm and weak SST anomalies over the CP, mainly due to the nonlinear zonal advection of the SST. Besides, a few quiescent periods are also observed in the simulations (e.g., years 1950-70), during which the value of $I_{\mathrm{wc}}$ goes below zero.

Figure 6 shows the Hovmöller diagrams for multiple intraseasonal and interannual fields from the last 100-yr simulation of the full model. Similar to Figs. 3 and 4, the wind bursts in Fig. $6 \mathrm{a}$ and the heating in Fig. $6 \mathrm{~b}$ mostly occur over the western and central Pacific. In particular, the extreme events (e.g., the one at year 1920) with strong wind bursts and heating anomalies are correlated with the warm SST anomalies, which is related to the modulation effects of the interannual SST on the intraseasonal multiplicative noise in Eq. (1). During the EP El Niño (e.g., the one at year 1950), the warm SST anomalies are first triggered by strong wind bursts over the WP, then move eastward along with deep thermocline depth anomalies, and eventually get intensified due to the thermocline feedback. In contrast, the CP El Niño (e.g., the one at year 1940) features warm SST anomalies in a much weaker magnitude over the $\mathrm{CP}$. Meanwhile, the small thermocline depth anomalies and weak thermocline feedback coefficient $\eta(x)$ indicate the less efficiency of the thermocline feedback for intensifying the SST anomaly over the CP. The SST budget analysis in Fig. $4 \mathrm{~g}$ confirms that the nonlinear zonal advection of the SST plays a major role in triggering CP El Niño. Besides, the eastward propagation of the downwelling waves following the WWBs can be seen in the zonal current and thermocline depth fields in Figs. 6d and 6e.

To further investigate the basic characteristics of the MJO and WWBs and their relationship with the ENSO, we pick three different type ENSO events (EP El Niño, CP El Niño, and La Niña) and show the Hovmöller diagrams for the intraseasonal zonal winds, MJO-band filtered zonal winds, and SST in Fig. 7. For the EP El Niño (see Figs. 7a-c), the easterly and westerly wind bursts are mostly prevalent over the western Pacific as the SST warms up, and then move eastward along with the warm water. The spatial scales of these wind bursts span from small-scale anomalies driven by the local noise to a 

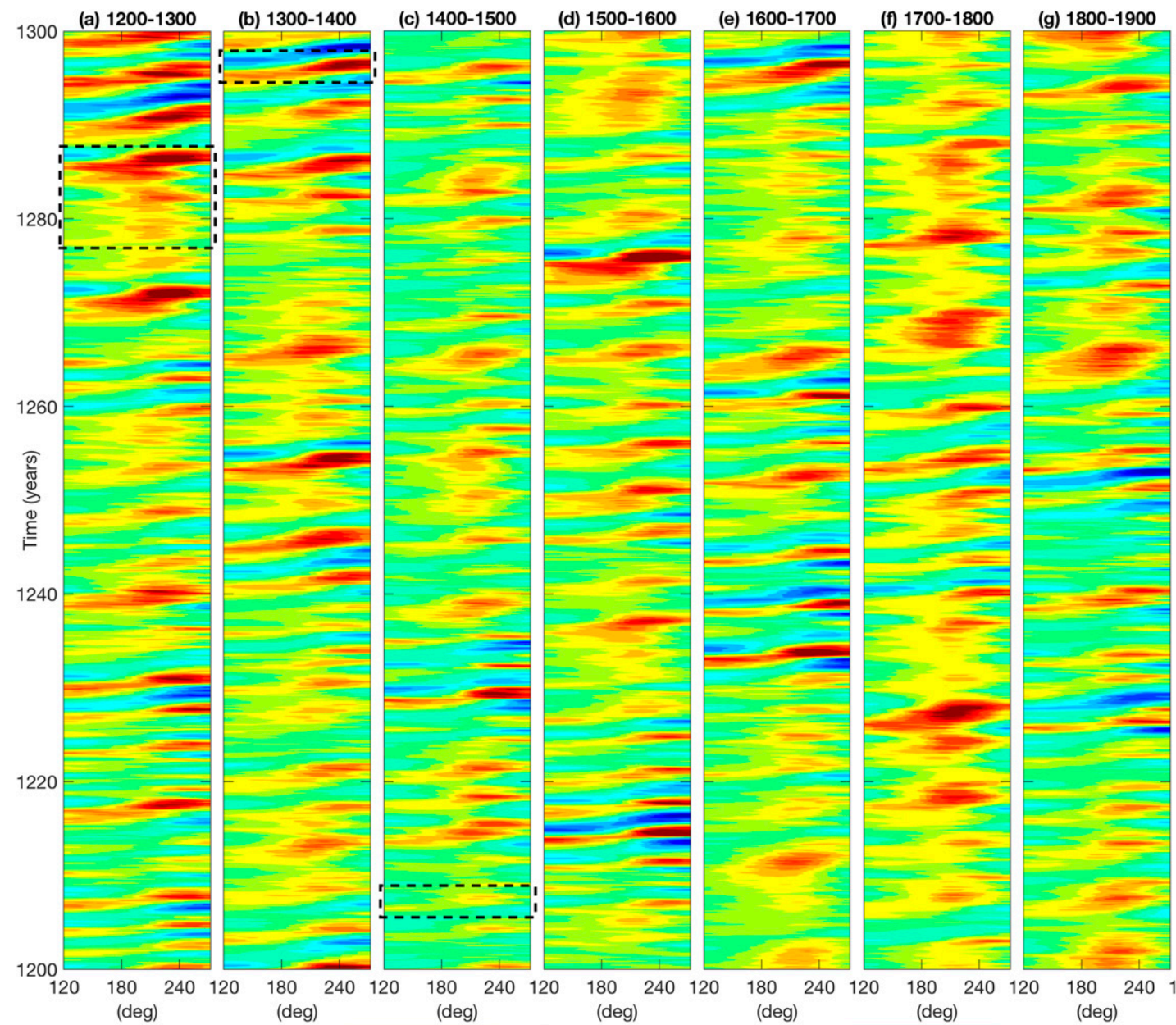

(h) $1900-2000$
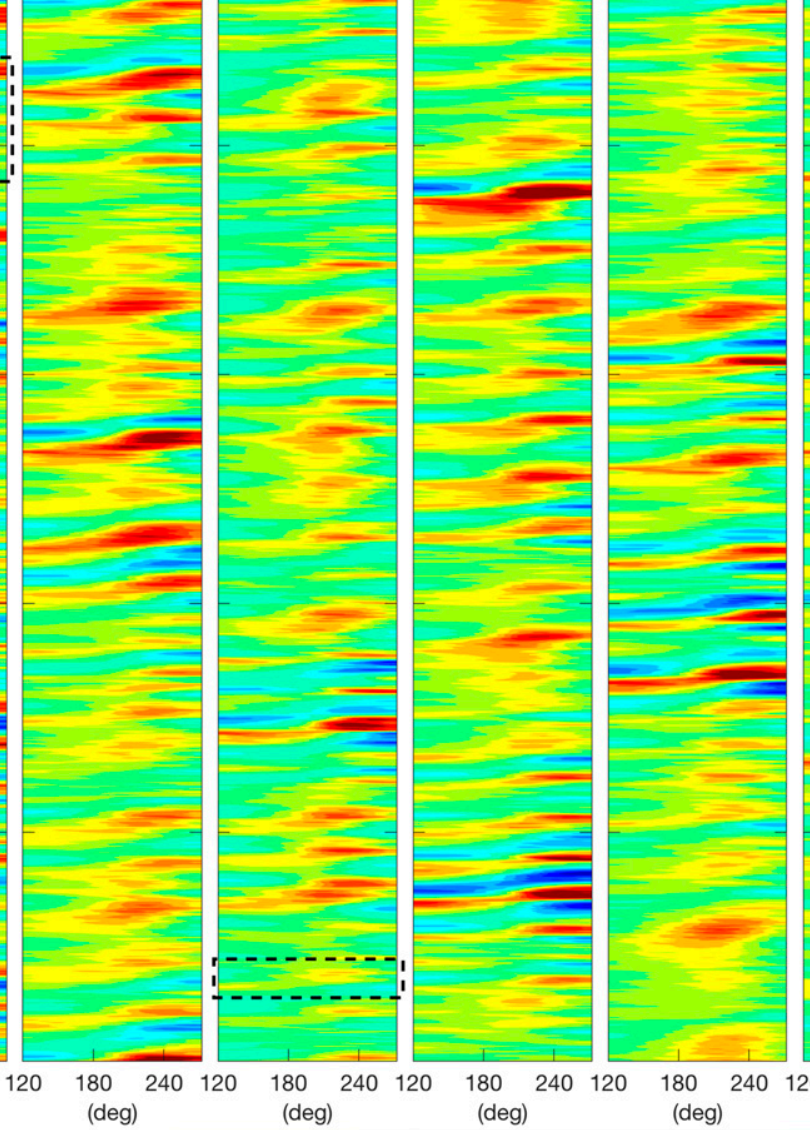

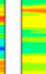
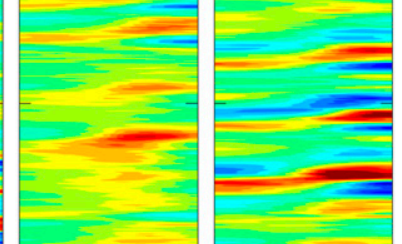

.
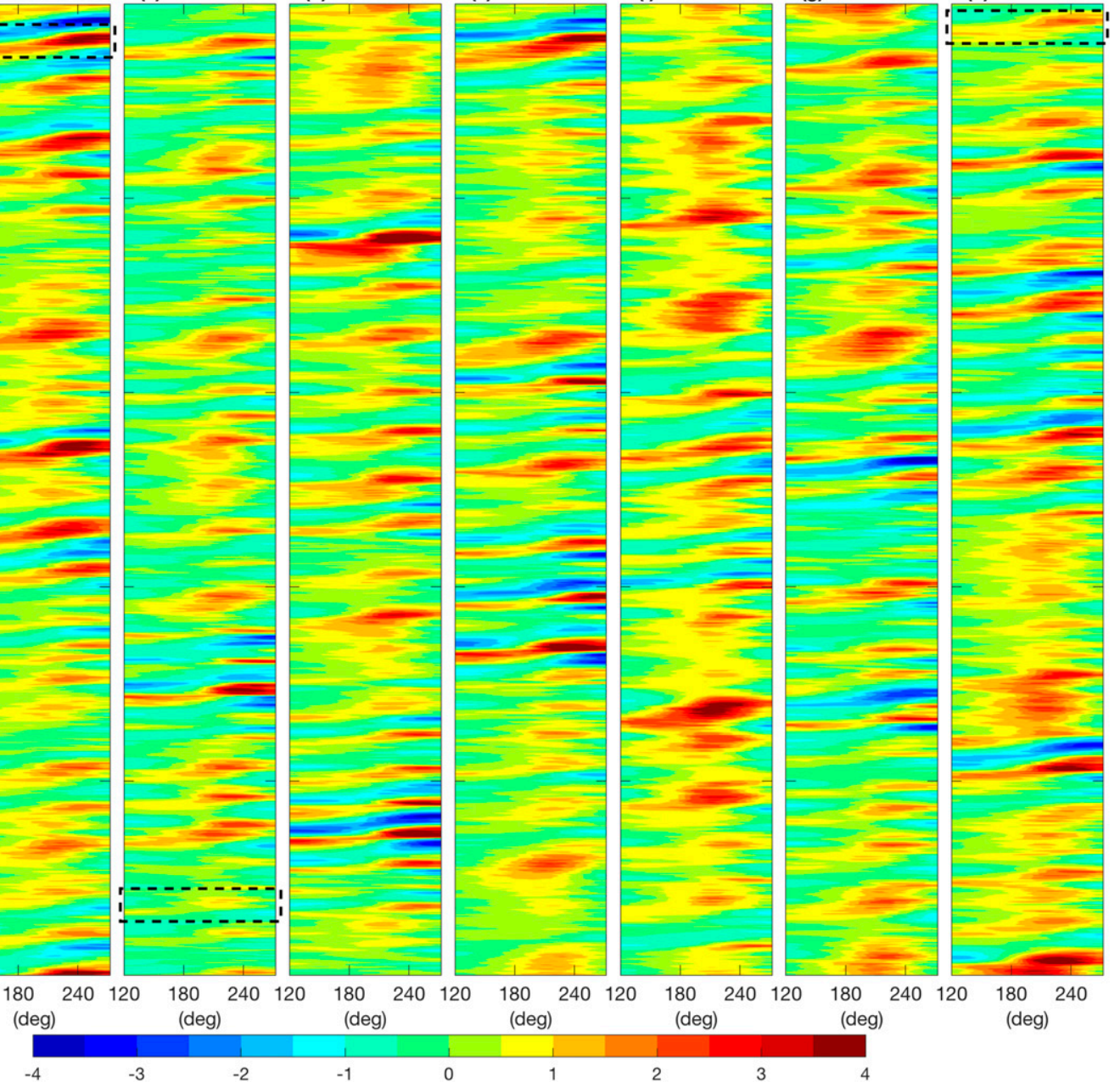

(deg)

$\begin{array}{ll}(\mathrm{deg}) & (\mathrm{deg})\end{array}$

0

2

3

(deg)

(deg)

FIG. 5. Hovmöller diagrams for equatorial SST (K) from the last 800-yr simulation of the full model. Each column includes a 100-yr simulation. The dashed boxes indicate different types of El Niño events that are directly compared with the observation in Fig. 9.

large-scale pattern up to one-third of the whole ocean domain. Consistent to the observation (Feng and Lian 2018), only part of these wind bursts can be explained by the MJO wind field that is also significant over the warm SST regions. In contrast to the EP El Niño, the CP El Niño in Figs. 7e-g features much weaker wind bursts and the MJO, which do not move eastward and are only confined over the western Pacific. Also, the magnitudes of wind bursts do not vary much before and after the $\mathrm{CP}$ El Niño, reflecting less modulation effects by the latter. During the La Niña in Figs. 7i-k, both wind bursts and the MJO are suppressed due to the cold SST. Essentially, these distinct behaviors of wind bursts and the MJO during different ENSO events should stem from the state-dependent intraseasonal noise. Figures $7 \mathrm{~d}, 7 \mathrm{~h}$, and 71 show the composite horizontal profiles of SST from these three different type ENSO events. The EP El Niño in Fig. 7d features a strong warm SST anomaly over the eastern Pacific and decays gradually as the latitude goes poleward. In contrast, the CP El Niño in Fig. $7 \mathrm{~h}$ is characterized by a weak SST anomaly over the CP. Notably, this westward displacement of warm SST centers in the model output is not as large as the observation (Capotondi et al. 2015), presumably due to the simplification of the model. The La Niña in Fig. 71 features a similar spatial pattern as the EP El Niño but with cold SST in a much weaker magnitude.

Figure 8a shows the time series of equatorial SST in three different Niño regions. Overall, all the time series exhibit irregular features on top of the oscillatory pattern, consistent with the observed ENSO events with significant event-to-event differences. Also, a clear phase relation exists among the peaks of these time series from Niño-4 to Niño-3.4 to Niño-3, reflecting the persistent eastward movement of the Niño. Besides, the maximum magnitude of warm SST anomalies is mostly larger than those of cold SST anomalies, resembling the observed amplitude asymmetry between El Niño and La Niña phases. In addition, there are rarely events that peak only at the Niño-4 region. Instead, almost all events span over the entire Niño regions, strongly implying that this model successfully captures ENSO irregularity, rather than only the two distinct 


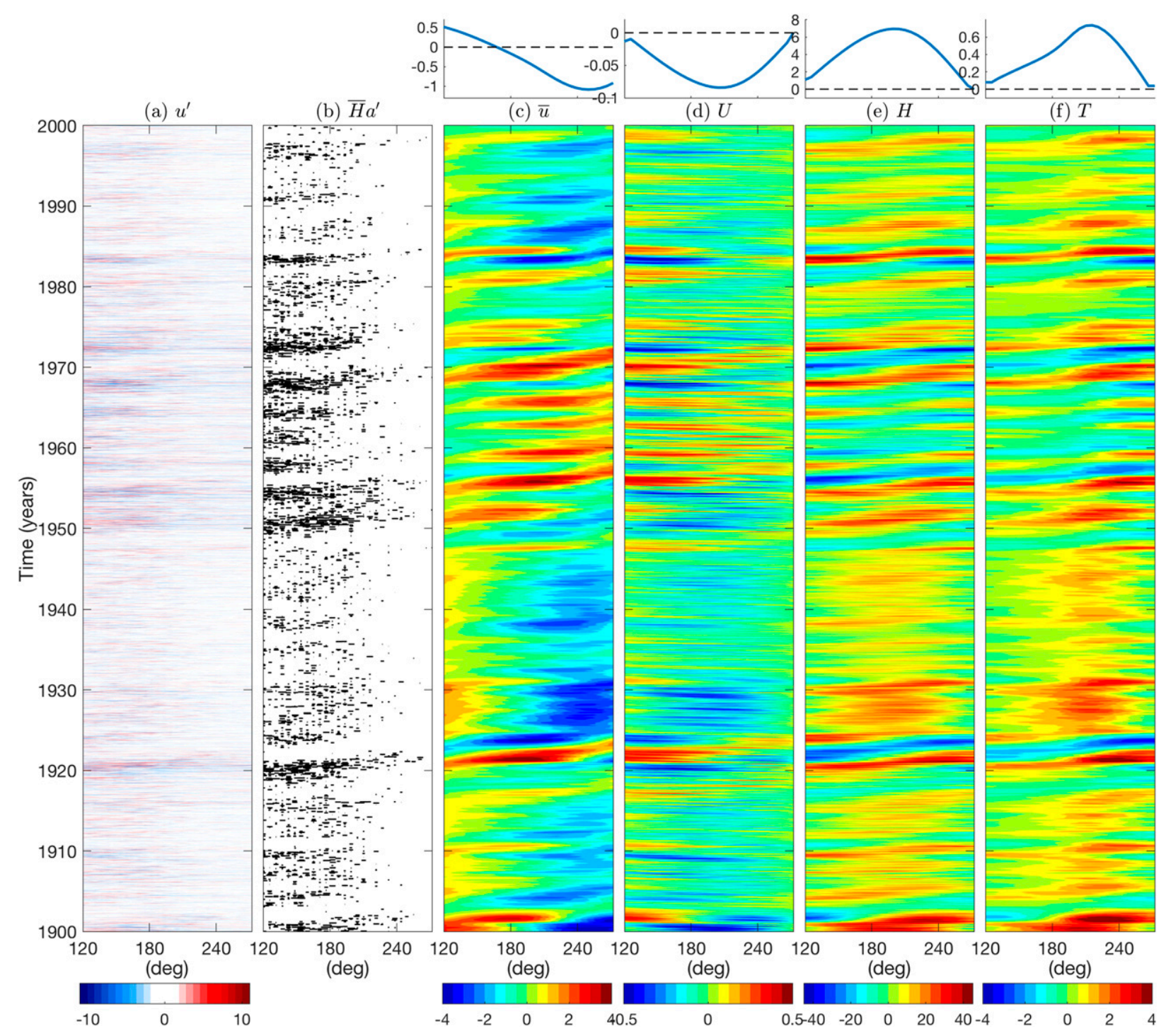

FIG. 6. Hovmöller diagrams for (a) intraseasonal zonal winds $u^{\prime}\left(\mathrm{m} \mathrm{s}^{-1}\right)$, (b) intraseasonal convective activity $\bar{H} a^{\prime}\left(\mathrm{K}\right.$ day $\left.{ }^{-1}\right)$, (c) interannual zonal winds $\bar{u}\left(\mathrm{~m} \mathrm{~s}^{-1}\right)$, (d) ocean current $U\left(\mathrm{~m} \mathrm{~s}^{-1}\right)$, (e) thermocline depth $H(\mathrm{~m})$, and (f) SST $T$ (K) from the last 100-yr simulation of the full model. The curves above (c)-(f) show the climatological mean of corresponding fields below. The black shading in (b) indicates $\bar{H} a^{\prime}$ anomalies that are larger than $4 \mathrm{~K}$. Units are dimensional.

types of El Niños. Here we further compare the model output with the observation in terms of the equilibrium PDF and power spectrum density of equatorial SST. Figures $8 \mathrm{~b}-\mathrm{d}$ show that the full model captures the realistic variability and nonGaussian features in different Niño regions. For the Niño-4 region in Fig. 8b, the model reproduces the variance of the observation, despite that the estimation of skewness and kurtosis contains errors. For the Niño-3.4 region in Fig. 8c, the equilibrium PDF of SST from the model and observation are quite similar to each other with comparable values of standard deviation, skewness, and kurtosis. The positive skewness indicates the significant amplitude asymmetry between El Niño and La Niña phases, and the large value of kurtosis indicates the large occurrence frequency of extreme El Niño events. For the Niño-3 region in Fig. 8d, besides the comparable standard deviation, the model also reproduces positive skewness and kurtosis greater than 3 , similar to the observation. In particular, the kurtosis of the Niño-3 becomes even larger than that of the Niño-3.4, resembling the observation that the extreme El Niño typically propagates to the farther east. Notably, the small difference in the PDFs is due to a slight underestimation of the quiescent events over the EP. Nevertheless, there still exist large discrepancies in the non-Gaussian characteristics of the ENSO between model output and the observation, presumably due to the simplification of this model. Specifically, although the model reproduces the right signs of the skewness over the Niño-3.4 and Niño-3 regions, its absolute value from the model output is only half of that from the observation. This 

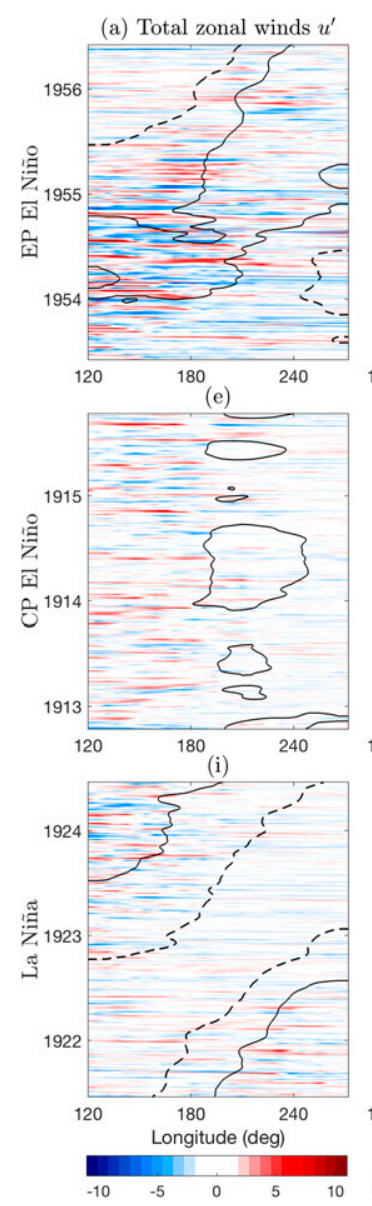
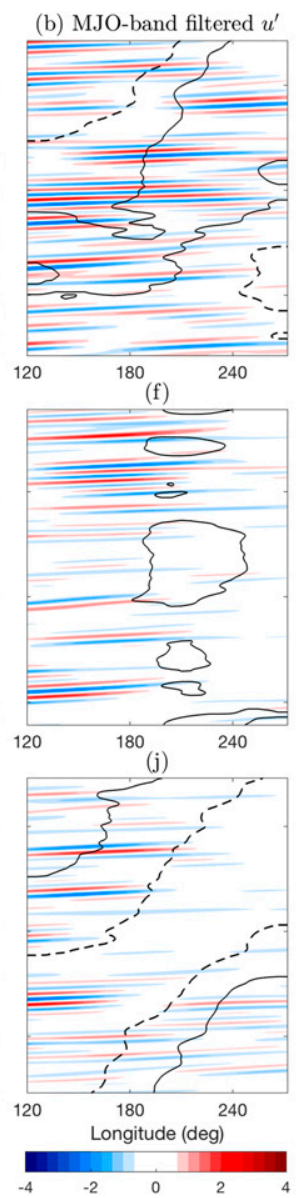

(c) SST
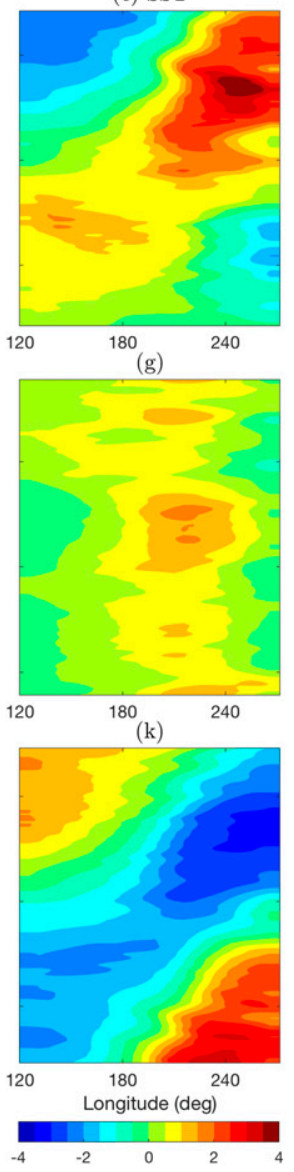

(d) Composite EP El Niño

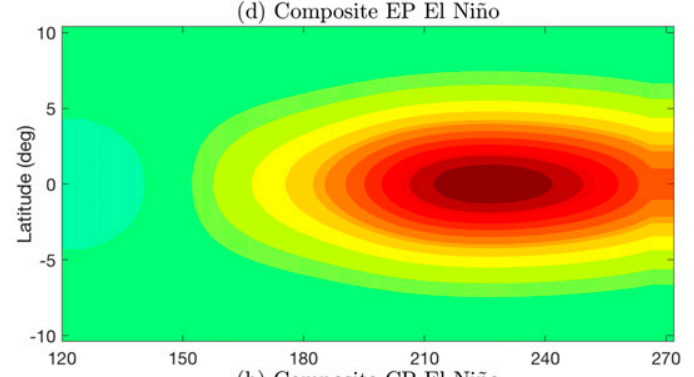

(h) Composite CP El Niño
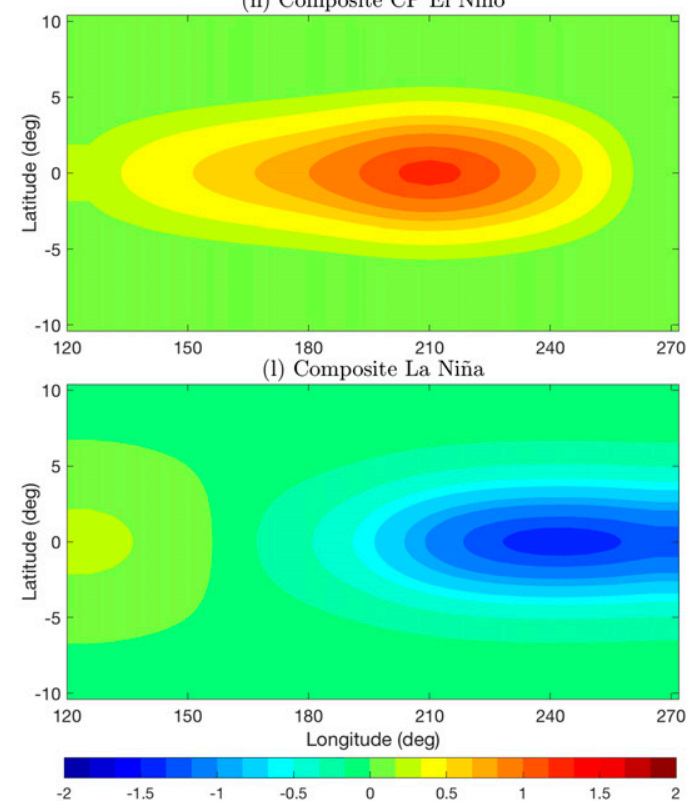

FIG. 7. (a)-(c) The Hovmöller diagrams for total intraseasonal zonal winds $u^{\prime}\left(\mathrm{m} \mathrm{s}^{-1}\right)$, MJO-band (wavenumber 1-5 and 30-90 day period) filtered $u^{\prime}\left(\mathrm{m} \mathrm{s}^{-1}\right)$, and equatorial SST (K) from a selected EP El Niño event. The solid and dashed contours in (a) and (b) indicate $1.0 \mathrm{~K}(-1.0 \mathrm{~K})$ SST, respectively. (e)-(g),(i)-(k) As in (a)-(c), but for CP El Niño and La Niña events. (d),(h),(l) Composite horizontal profiles of SST from different types of ENSO events, based on the last 1800-yr model output. Similar ENSO events are categorized as the same type according to the three Niño indexes (EP El Niño: Niño-3.4 index > 1.0 K, Niño-3 index > 1.0 K; CP El Niño: Niño-4 index > $0.5 \mathrm{~K}, 0.5 \mathrm{~K}<\mathrm{Niño-3}$ index $<1.0 \mathrm{~K}$; La Niña: Niño-3.4 index $<0.5 \mathrm{~K})$.

discrepancy can be attributed to the simplified form of the state-dependent intraseasonal noise so that the amplitude asymmetry between El Niño and La Niña phases in the model is not as significant as the observation. As for the power spectrum density, the clear 2-7 year spectrum band with a dominant 5 -yr peak is found in these three Niño regions in Figs. 8e-g, resembling the typical period of the ENSO events. However, the interannual spectrum peak of the Niño-4 that appears in the simulation is not seen in the observation, presumably due to the simplified atmosphere-ocean coupled dynamics over this region.

The simulation in a long period reproduces different types of ENSO events with significant event-to-event differences. Figures 9a-d show the Hovmöller diagrams for equatorial SST during four different ENSO episodes from the observation, including delayed super El Niño in 2014-16 (Fig. 9a), CP El Niño in 2009-10 (Fig. 9b), 5-yr CP El Niño followed by a super El Niño in the 1990s (Fig. 9c), and super El Niño in 1982-83 (Fig. 9d). Here we pick four similar ENSO events in Figs. 9e-h from the last 800-yr simulation of the full model (see Fig. 5). To illustrate the underlying mechanisms responsible for these ENSO episodes, we also show the corresponding wind bursts in Figs. 9i-1, the nonlinear zonal advection in Figs. 9m-p, and the thermocline feedback in Figs. 9q-t. Specifically, the delayed moderate El Niño in Fig. 9e is preceded by warm SST anomalies over the western and central Pacific in the first year and then followed by an extreme El Niño with strong warm SST anomalies over the EP in the second year. As for wind bursts in Fig. 9i, the first WWB tends to trigger an EP Niño in the first year but a series of strong follow-up easterly wind bursts terminates it. In the second year, a sequential WWBs are only followed by weak easterly wind events and thus trigger a strong El Niño. This event is qualitatively similar to the 2014-16 delayed El Niño event. The thermocline feedback in Fig. 9q plays a dominant role in intensifying the warm SST anomalies, while the nonlinear zonal advection in Fig. $9 \mathrm{~m}$ induces their westward displacement. The CP El Niño in Fig. 9f features a weak warm SST anomaly over the CP, 

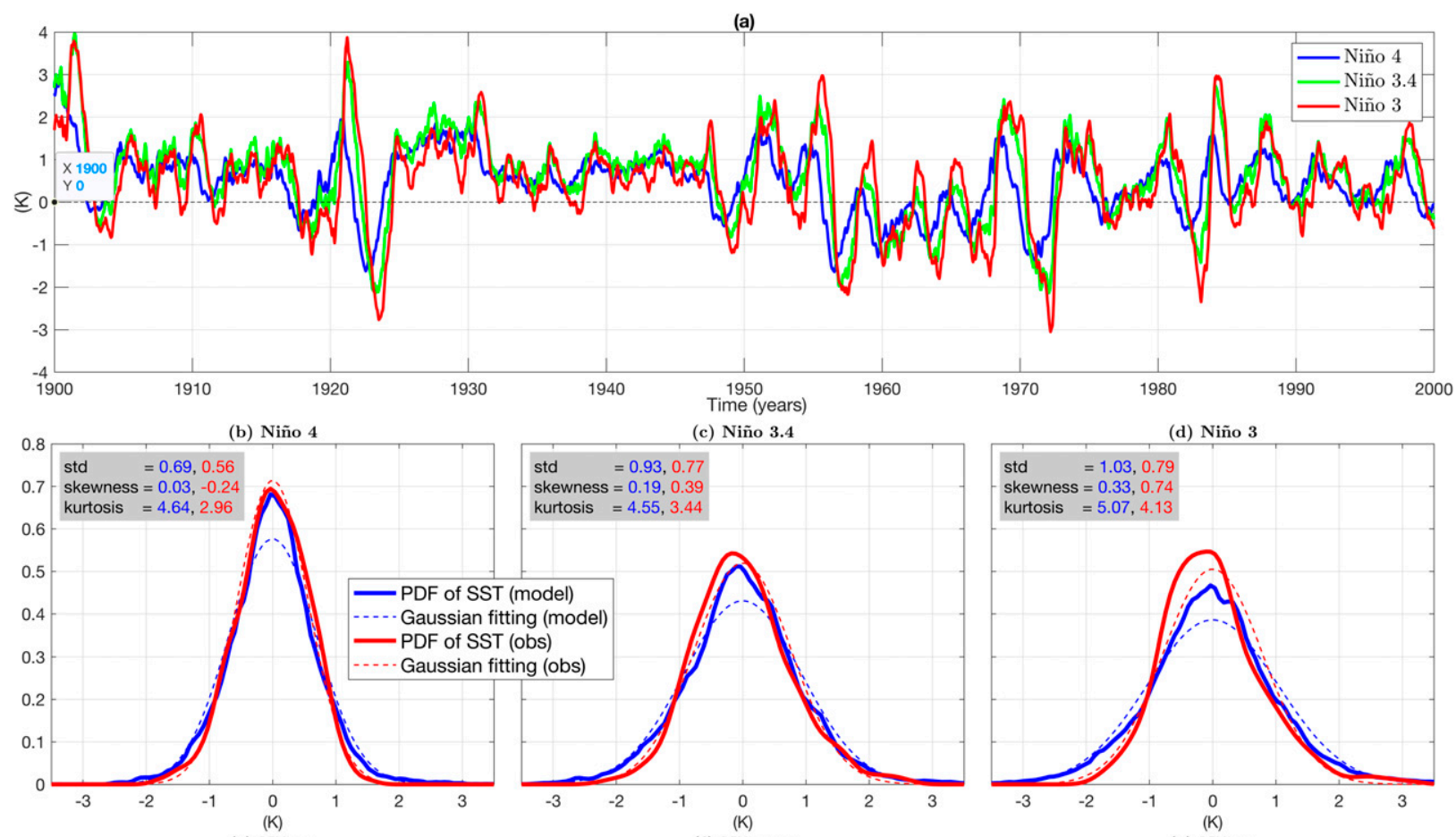

(c) Niño 3.4

(d) Niño 3

(e) Niño 4
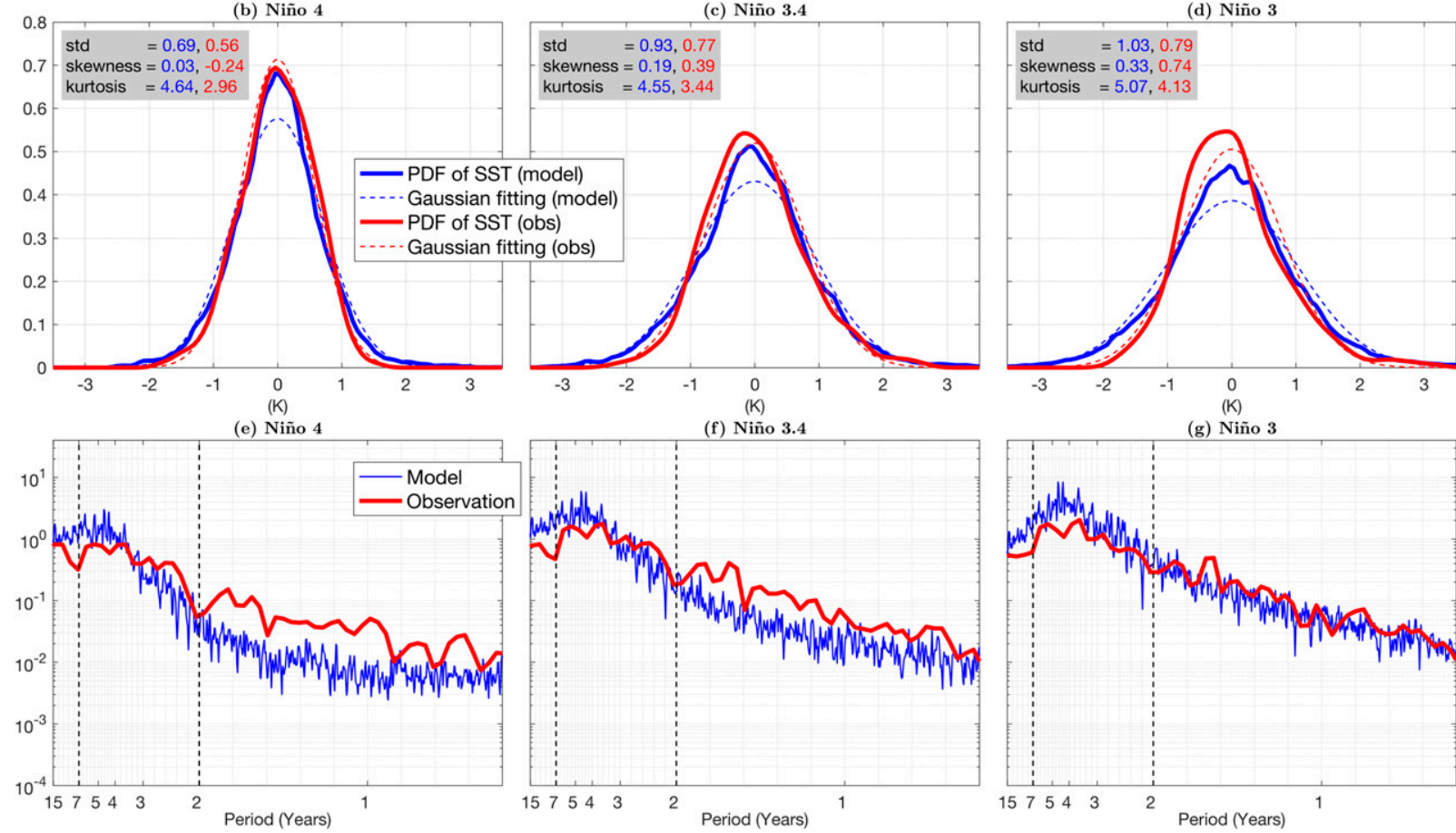

(f) Niño 3.4

(g) Niño 3
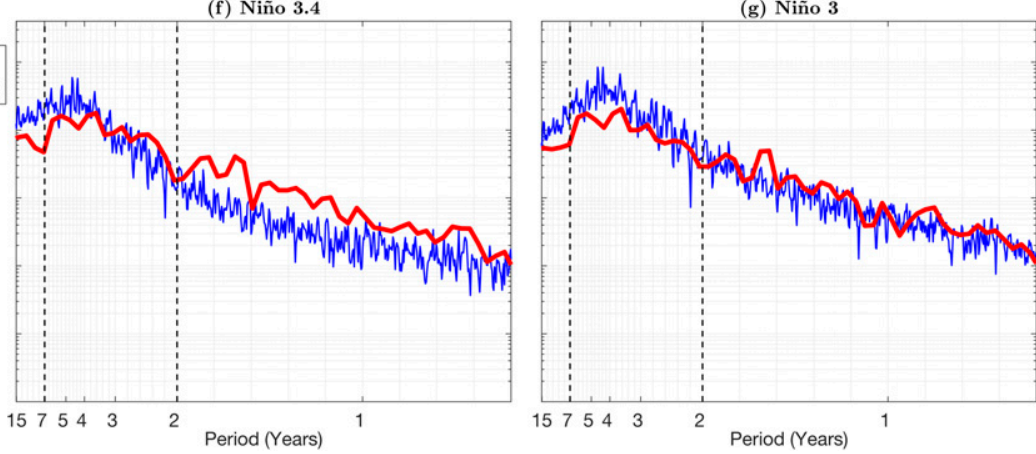

FIG. 8. (a) The time series of equatorial SST (K) in three different El Niño regions (Niño-4, Niño-3.4, and Niño-3) from the last 100-yr simulation of the full model. (b) The equilibrium PDF of equatorial SST in the Niño-4 region, where the blue curve is based on the last 1800-yr model output and the red curve is based on the 150-yr HadISST observational data. The dashed curves are the corresponding Gaussian distribution fitting. The second, third, and fourth statistical moments are summarized in the gray box of each panel (blue for model and red for observation). (e) The log-scale power spectrum density of equatorial SST in the Niño-4 region. The two vertical dashed lines indicate typical El Niño period of 2-7 years. (c),(f),(d),(g) As in (b) and (e), but for equatorial SST in the Niño-3.4 [in (c) and (f)] and Niño-3 [in (d) and (g)] regions.

slightly to the east of the observed one in Fig. 9b. This CP El Niño is first triggered by significant wind bursts in Fig. 9j over the WP and then intensified over the $\mathrm{CP}$ due to the nonlinear zonal advection in Fig. 9n, while the thermocline feedback is negligible. Besides, the full model reproduces the observed 1990s episode in Fig. 9g, during which easterly/westerly wind bursts prevail over the WP and both effects have a comparable contribution to the El Niño over the central and eastern Pacific. Last, the model also captures an extreme El Niño with large SST anomalies in Fig. 9h, mainly due to the significant wind bursts across the Pacific and thermocline feedback over the EP. To sum up, these different types of ENSO events are first triggered by easterly/westerly wind bursts over the WP, moving eastward along with warm SST anomalies. The nonlinear zonal advection of SST plays an essential role in inducing the CP El Niño in the presence of the background Walker circulation, while the thermocline feedback leads to the EP El Niño in a strong magnitude. Although the nonlinear zonal advection term seems significant in all events (see Figs. $9 \mathrm{~m}-$ p), there is a fundamental difference about its role in inducing the EP and CP Niño. In fact, Thual et al. (2018) showed that the model can still reproduce the EP Niño in the absence of the nonlinear zonal advection. In contrast, both nonlinear zonal advection and the background easterlies are found to play a key role in inducing the CP Niño (Chen and Majda 2016). 


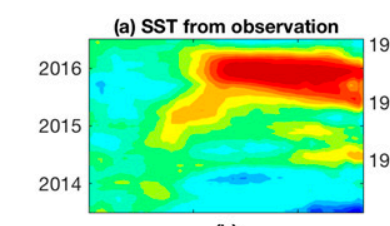

(b)

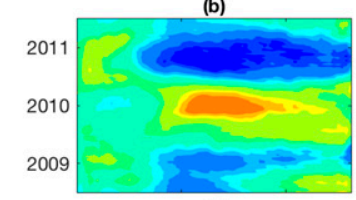

(c)
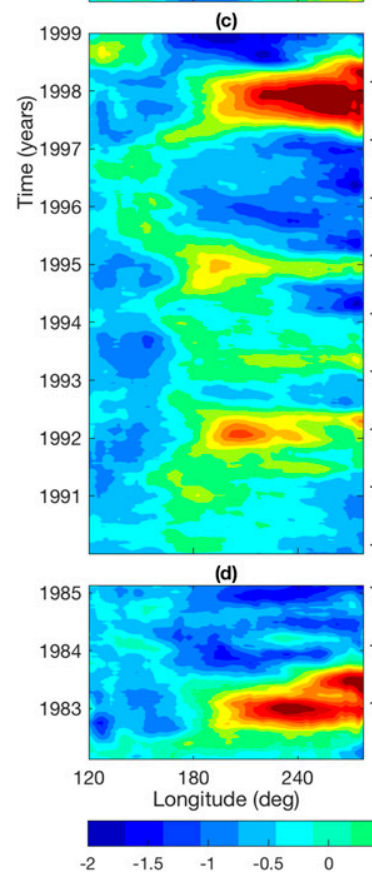

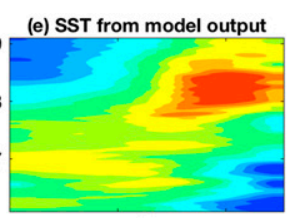

(f)

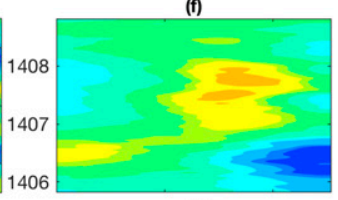

(g)

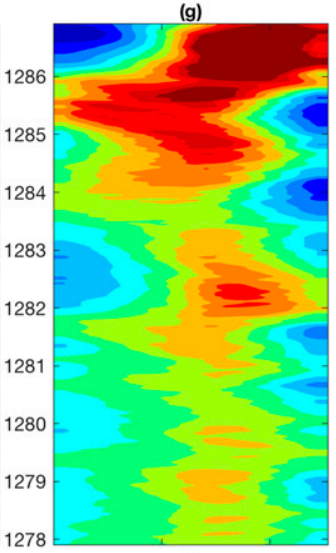

(h)

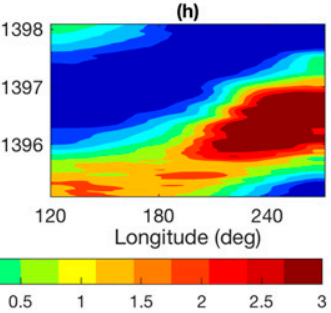

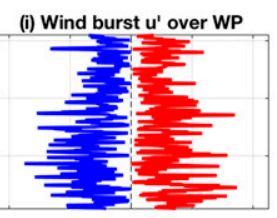
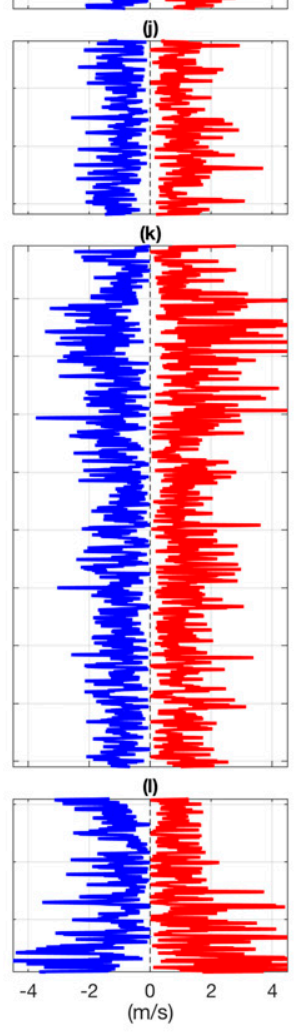

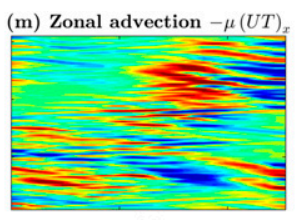

(n)

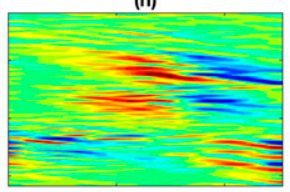

(o)

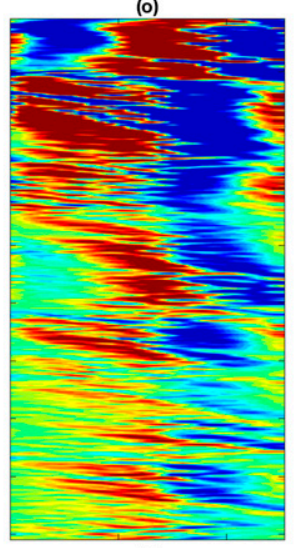

(p)

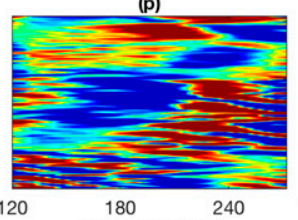

Longitude (deg)

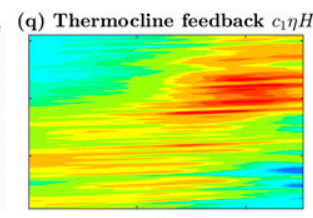

(r)

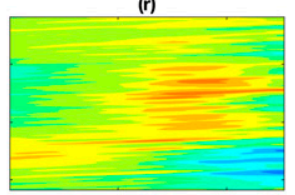

(s)

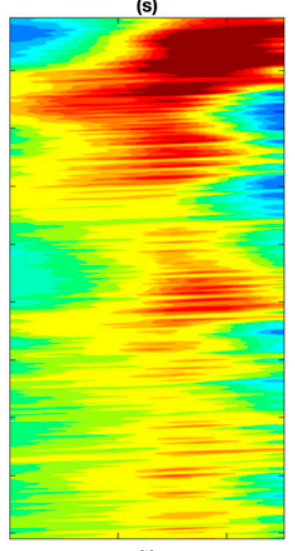

(t)

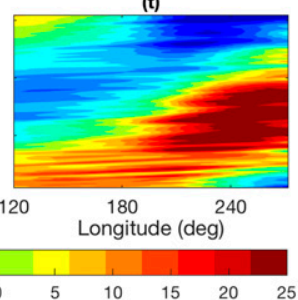

FIG. 9. The Hovmöller diagrams for equatorial SST (K) during four different types of El Niño events from the observation, including (a) delayed super El Niño in 2014-16, (b) CP El Niño in 2009-10, (c) 5-yr CP El Niño followed by a super El Niño in 1990s, and (d) super El Niño in 1982-83. In (e)-(h), four events that are chosen from the last 800-yr simulation of the full model share many similarities with the observed ones in (a)-(d). The Hovmöller diagrams for (i)-(l) intraseasonal wind bursts $u^{\prime}\left(\mathrm{m} \mathrm{s}^{-1}\right),(\mathrm{m})-(\mathrm{p})$ the nonlinear zonal advection term $-\mu(U T)_{x}\left(\mathrm{~K} \mathrm{yr}^{-1}\right)$, and (q)-(t) the thermocline feedback term $c_{1} \eta H\left(\mathrm{~K} \mathrm{yr}^{-1}\right)$ during these four events from the model output. Panels in each column share the color bar at the bottom. The red (blue) curve in (i)-(l) shows the time series of root-mean-square of westerlies (easterlies) over the WP, which is further smoothed by taking moving mean over 5 days. Units are dimensional.

\section{Concluding discussion}

Capturing the ENSO diversity with all different types of ENSO events occurring in a realistic frequency is a challenging problem for present-day GCMs. The goal of this study is to obtain a simple stochastic dynamic model that captures realistic features of the ENSO, providing useful guidelines for improving ENSO simulations in GCMs. In this paper, a tropical stochastic skeleton modeling framework is developed to simulate the realistic interactions among wind bursts and the MJO, the El Niño, and the background Walker circulation. Specifically, we considered state-dependent noise in the intraseasonal atmosphere to trigger extreme and delayed El Niños and capture the observed non-Gaussian and irregular features of SST in different Niño regions. Meanwhile, we added the nonlinear zonal advection of SST in the presence of the background Walker circulation to reproduce the occurrence of the CP El Niño. Also, we introduced a simple stochastic equation for governing the multidecadal variation of the Walker circulation, modulating the switching between EP and CP El Niño regimes. Last, we compared the model output with the observation and concluded that the full model successfully simulates the individual El Niño episodes and their underlying mechanisms.

The intraseasonal skeleton model for the MJO (Majda and Stechmann 2009) was improved here by adding the multiplicative noise in moisture and convective activity. This statedependent noise accounts for the modulation effect by the interannual and multidecadal variation of large-scale fields, resulting in the non-Gaussian features of the ENSO and the amplitude asymmetry between the El Niño and La Niña phases. That said, the model-simulated skewness of Niño-3.4 and 
Niño-3 is smaller than the observation, indicating that other important physical processes also contribute to the non-Gaussian features of the ENSO in nature. Such a simple stochastic intraseasonal model should be useful for modeling wind bursts when discussing the impact of westerlies and easterlies on the ENSO. For example, Lian et al. (2014) and Chen et al. (2015) added a set of idealized WWBs in an intermediate oceanatmosphere coupled model to investigate the effects of WWBs on the El Niño diversity. A series of papers by Hu et al. (2014), Fedorov et al. (2015), and $\mathrm{Hu}$ and Fedorov (2016, 2019) studied the effects of both easterly and westerly wind bursts on the El Niño development by superimposing the observed wind bursts onto a state-of-art climate model. In contrast, the intraseasonal skeleton model has the advantage in explicitly resolving the wind bursts under the modulation effects of ENSO fields, providing a dynamical way to superimpose westerly and easterly wind bursts. Besides, the model demonstrates that a large portion of wind bursts in this model is from the $\mathrm{MJO}$, taking into account the significant impact of the MJO on the ENSO dynamics. However, only part of the simulated WWBs can be attributed to the MJO because the intraseasonal skeleton model also includes another three wave modes besides the MJO mode. Also, the spectral peaks of the simulated MJO extend from wavenumber 2 to 10 , differing from the observed MJO band in wavenumbers 1-3. Although the relationship between WWBs and the MJO is still under debate (Fasullo and Webster 2000; Seiki and Takayabu 2007a), recent studies showed that only about one-third of WWBs can be explained by the MJO in observations and CMIP5 models (Feng and Lian 2018). This fraction value of WWBs only increases to $57 \%$ even in the case that WWBs and the MJO are defined by overlapping frequency bands (Puy et al. 2016). Thus it is worth investigating further whether the MJO is the main driver of WWBs and how they impact on the ENSO dynamics based on the observation.

Despite its simple formalism, this full model proposes a physical mechanism that should be potentially useful for explaining the observed ENSO diversity. Traditional intermediate models typically describe the ENSO dynamics by an oscillator theory, resulting in few types of ENSO events in a regular oscillatory pattern. As for GCMs, C. Chen et al. (2017) diagnosed various model biases in the CMIP5 models on the statistical quantities of ENSO dynamics, including the seasonal phase locking, diversity in peak location, and propagation direction as well as the El Niño-La Niña asymmetry in amplitude, duration, and transition. In contrast, this stochastic model realistically reproduces the irregular pattern of the ENSO, accounting for significant event-to-event differences as observed in nature. Meanwhile, the full model realistically captures the non-Gaussian statistical features of SST in different Niño regions, including the positive skewness for the amplitude asymmetry between El Niño and La Niña phases and the large value of kurtosis for the occurrence of extreme El Niños. Besides, it reproduces the realistic occurrence frequency of different types of ENSO events in the 2-7-yr period that is akin to the observation. Here we propose this full model as one possible mechanism for explaining the observed ENSO diversity in nature, complementary to other physical mechanisms that have been studied in the literature (Capotondi et al. 2015). For example, the numerical simulations by Lengaigne et al. (2004) and Fedorov et al. (2015) demonstrated that the tropical Pacific Ocean background conditions play an important role in determining the type of El Niño through the upper-ocean heat content. Besides, off-equatorial atmospheric variability such as the North Pacific Oscillation-related wind anomalies has been shown to trigger different types of El Niño through thermodynamic coupling (Larson and Kirtman 2013) and dynamically energizing equatorial processes ( $\mathrm{Yu}$ and Kim 2011).

However, there still exists a difference over the Niño-3 region between the simulation and observation, which may well be more than the state-dependent intraseasonal noise. In detail, the El Niño variability range and intensity differ significantly between the simulation and observation in Fig. 8d. According to Fig. $8 \mathrm{~g}$, it appears that the 2-7-yr time-scale dynamics from the simulation is amplified in comparison to the observation, while their higher frequency processes in this region match well. In other words, this stochastic model has a more uniformly noisy spectral Niño-3 response with the dominant and forced 2-7-yr ENSO response mode being amplified. It is clear that there are more frequency structures in the observed than that resolved in the model, so it will be helpful to consider a combination of oscillator and stochastic representation models to test the distinction between exciting specific periodic type modes versus a generic stochastic excitation of the full 2-7-yr power spectrum. For example, Bruun et al. (2017) used a highly accurate method called dominant frequency state analysis (DFSA) to provide evidence of stable ENSO features and concluded the existence of long-term and short-term climatic cycles of the ENSO process with multiple resonance frequencies. It should be an interesting research topic to use such a method to analyze the signal to noise properties in this nonlinear process model and explain the discrepancy in Fig. 8d.

In addition, the full model demonstrates the crucial role of easterly/westerly wind bursts and the MJO, the nonlinear zonal advection, and the thermocline feedback in generating the El Niño events, providing useful guidelines for improving the ENSO simulation in the GCMs. Wind bursts are simulated by the intraseasonal skeleton model with the multiplicative noises, pointing out the necessity of capturing the state-dependent wind bursts and the MJO in the ENSO simulations. In contrast to the traditional ENSO theory, the nonlinear zonal advection of SST in this full model considers a total SST flux from both background and anomalous fields with no ad hoc parameterization of background SST gradient. This highlights the necessity for better resolving the zonal advection effect and the eastward displacement of the warm pool edge during the CP El Niño in GCMs. Last, the full model describes the dynamic interactions among winds bursts and the MJO, the El Niño, and the background Walker circulation, suggesting the importance of the coupling processes from intraseasonal to interannual to multidecadal scales. Also, it is worth pointing out that this model is a fully coupled atmosphereocean model, where winds drive ocean currents through wind stress and the resulting SST anomalies feedback on atmospheric dynamics through surface heat flux. Therefore, when 
the Walker circulation is built up due to the imbalanced external source of cooling and moistening, other oceanic background states such as the mean SST gradient are simultaneously induced. In fact, many previous studies have shown that oceanic background states play an important role in modulating ENSO dynamics (Capotondi et al. 2015; Puy et al. 2019b). For example, the SST budget analysis based on a long-term GCM simulation indicates that the low-frequency modulation of ENSO is correlated with the equatorial zonal SST gradient changes in the Pacific decadal variability cycle (Imada and Kimoto 2009).

The full model in the current form can be elaborated and extended in various ways. For example, the SST field is projected only onto the leading symmetric parabolic cylinder function that reaches the maximum in the tropics and decays on both sides. It should be interesting to allow more symmetric and antisymmetric meridional modes to take account for the asymmetric characteristics of the ENSO, particularly during its mature phase with a southward shift of the maximum wind stress (Vecchi 2006). Another interesting research direction is to add the ENSO synchronization to the seasonal cycle by incorporating a cloud radiative feedback for the marked seasonal variations of convective activity and cloud cover over the EP (Thual et al. 2017). It is also worth exploring the long-term statistics of the MJO and ENSO simulations as well as the longterm changes in the background Walker circulation, a highpriority research topic under the climate change. Besides, due to the simplicity of this model framework, almost all the simulated El Niño events are originated from the western basin and propagate eastward, in contrast to the observation that some EP Niño events propagate westward. Thus it should be also interesting to include the westward-propagating mode, which is prominent when the background state has a shallow thermocline as described by Fedorov and Philander (2000). Also, almost no CP La Niñas were simulated in this model, presumably due to the simplification assumptions and physical parameter choices. Last, the budget analysis of the observed mixed layer heat by $\mathrm{Su}$ et al. (2010) found that the nonlinear zonal temperature advection is essential in the far eastern Pacific, different from the result here. Thus, the importance of the nonlinear zonal advection of SST for CP El Niño genesis should be further verified by using observational datasets under this modeling framework. This study should also benefit from a detailed comparison about the background Walker circulation with the observation.

Acknowledgments. This research of A.J.M. is partially supported by the office of Naval Research ONR MURI N0001412-1-0912 and the Center for Prototype Climate Modeling (CPCM) in New York University Abu Dhabi (NYUAD) Research Institute. Q.Y. is funded as a postdoctoral fellow by CPCM in NYUAD Research Institute. The research of N.C. is supported by the Office of Vice Chancellor for Research and Graduate Education (VCRGE) at University of WisconsinMadison. We thank Sulian Thual for providing the original code of the TSS model.

Data availability statement. The first SST data used in this study are from the OISST reanalysis project and downloaded from its website (https://www.ncdc.noaa.gov/oisst). The second SST data are from the Met Office HadISST dataset and downloaded from its website (https://www.metoffice.gov.uk/hadobs/ hadisst/). The code and model output of all experiments are available on application to the corresponding author.

\section{REFERENCES}

An, S.-I., and F.-F. Jin, 2001: Collective role of thermocline and zonal advective feedbacks in the ENSO mode. J. Climate, 14, 3421-3432, https://doi.org/10.1175/1520-0442(2001)014<3421: CROTAZ $>2.0 . \mathrm{CO} ; 2$.

— Y.-G. Ham, J.-S. Kug, F.-F. Jin, and I.-S. Kang, 2005: El NiñoLa Niña asymmetry in the Coupled Model Intercomparison Project simulations. J. Climate, 18, 2617-2627, https://doi.org/ 10.1175/JCLI3433.1.

Ashok, K., S. K. Behera, S. A. Rao, H. Weng, and T. Yamagata, 2007: El Niño Modoki and its possible teleconnection. J. Geophys. Res., 112, C11007, https://doi.org/10.1029/2006JC003798.

Averina, T., and S. Artemiev, 1988: Numerical solution of systems of stochastic differential equations. Russ. J. Numer. Anal. Math. Model., 3, 267-286, https://doi.org/10.1515/rnam.1988.3.4.267.

Barnston, A. G., M. K. Tippett, M. L. L'Heureux, S. Li, and D. G. DeWitt, 2012: Skill of real-time seasonal ENSO model predictions during 2002-11: Is our capability increasing? Bull. Amer. Meteor. Soc., 93, 631-651, https://doi.org/10.1175/ BAMS-D-11-00111.1.

Battisti, D. S., 1988: Dynamics and thermodynamics of a warming event in a coupled tropical atmosphere-ocean model. J. Atmos. Sci., 45, 2889-2919, https://doi.org/10.1175/15200469(1988)045<2889:DATOAW>2.0.CO;2.

- and A. C. Hirst, 1989: Interannual variability in a tropical atmosphere-ocean model: Influence of the basic state, ocean geometry and nonlinearity. J. Atmos. Sci., 46, 1687-1712, https:// doi.org/10.1175/1520-0469(1989)046<1687:IVIATA>2.0.CO;2.

Bellenger, H., É. Guilyardi, J. Leloup, M. Lengaigne, and J. Vialard, 2014: ENSO representation in climate models: From CMIP3 to CMIP5. Climate Dyn., 42, 1999-2018, https:// doi.org/10.1007/s00382-013-1783-z.

Bjerknes, J., 1969: Atmospheric teleconnections from the equatorial Pacific. Mon. Wea. Rev., 97, 163-172, https://doi.org/ 10.1175/1520-0493(1969)097<0163:ATFTEP > 2.3.CO;2.

Bouche, D., J.-M. Ghidaglia, and F. P. Pascal, 2011: Error estimate for the upwind finite volume method for the nonlinear scalar conservation law. J. Comput. Appl. Math., 235, 5394-5410, https://doi.org/10.1016/j.cam.2011.05.050.

Bruun, J. T., J. I. Allen, and T. J. Smyth, 2017: Heartbeat of the Southern Oscillation explains ENSO climatic resonances. J. Geophys. Res. Oceans, 122, 6746-6772, https://doi.org/ 10.1002/2017JC012892.

Cai, J., J. Xu, Z. Guan, and A. M. Powell, 2019: Interdecadal variability of El Niño onset and its impact on monsoon systems over areas encircling the Pacific Ocean. Climate Dyn., 52, 7173-7188, https://doi.org/10.1007/s00382-016-3377-z.

Cai, W., and Coauthors, 2014: Increasing frequency of extreme El Niño events due to greenhouse warming. Nat. Climate Change, 4, 111-116, https://doi.org/10.1038/nclimate2100.

Cane, M. A., and S. E. Zebiak, 1985: A theory for El Niño and the Southern Oscillation. Science, 228, 1085-1087, https://doi.org/ 10.1126/science.228.4703.1085.

Capotondi, A., and Coauthors, 2015: Understanding ENSO diversity. Bull. Amer. Meteor. Soc., 96, 921-938, https://doi.org/ 10.1175/BAMS-D-13-00117.1. 
Chen, C., M. A. Cane, A. T. Wittenberg, and D. Chen, 2017: ENSO in the CMIP5 simulations: Life cycles, diversity, and responses to climate change. J. Climate, 30, 775-801, https://doi.org/ 10.1175/JCLI-D-15-0901.1.

Chen, D., and Coauthors, 2015: Strong influence of westerly wind bursts on El Niño diversity. Nat. Geosci., 8, 339-345, https:// doi.org/10.1038/ngeo2399.

Chen, L., T. Li, B. Wang, and L. Wang, 2017: Formation mechanism for 2015/16 super El Niño. Sci. Rep., 7, 2975, https:// doi.org/10.1038/s41598-017-02926-3.

Chen, N., and A. J. Majda, 2016: Simple dynamical models capturing the key features of the central Pacific El Niño. Proc. Natl. Acad. Sci. USA, 113, 11732-11737, https://doi.org/ 10.1073/pnas.1614533113.

- and - 2017: Simple stochastic dynamical models capturing the statistical diversity of El Niño Southern Oscillation. Proc. Natl. Acad. Sci. USA, 114, 1468-1473, https://doi.org/ 10.1073/pnas.1620766114.

$\longrightarrow$, and - 2020: Efficient nonlinear optimal smoothing and sampling algorithms for complex turbulent nonlinear dynamical systems with partial observations. J. Comput. Phys., 410, 109381, https://doi.org/10.1016/j.jcp.2020.109381.

— _ - and S. Thual, 2018: Observations and mechanisms of a simple stochastic dynamical model capturing El Niño diversity. J. Climate, 31, 449-471, https://doi.org/10.1175/JCLI-D16-0880.1.

Chen, S. S., R. A. Houze Jr., and B. E. Mapes, 1996: Multiscale variability of deep convection in relation to large-scale circulation in TOGA COARE. J. Atmos. Sci., 53, 1380-1409, https:// doi.org/10.1175/1520-0469(1996)053<1380:MVODCI >2.0.CO;2.

Clarke, A. J., 2008: An Introduction to the Dynamics of El Niño and the Southern Oscillation. Elsevier, 324 pp.

Dai, A., and T. Wigley, 2000: Global patterns of ENSO-induced precipitation. Geophys. Res. Lett., 27, 1283-1286, https:/doi.org/ 10.1029/1999GL011140.

Dewitte, B., S.-W. Yeh, and S. Thual, 2013: Reinterpreting the thermocline feedback in the western-central equatorial Pacific and its relationship with the ENSO modulation. Climate Dyn., 41, 819-830, https://doi.org/10.1007/s00382-012-1504-z.

Dong, B., R. T. Sutton, and A. A. Scaife, 2006: Multidecadal modulation of El Niño-Southern Oscillation (ENSO) variance by Atlantic Ocean sea surface temperatures. Geophys. Res. Lett., 33, L08705, https://doi.org/10.1029/2006GL025766.

Fasullo, J., and P. J. Webster, 2000: Atmospheric and surface variations during westerly wind bursts in the tropical western Pacific. Quart. J. Roy. Meteor. Soc., 126, 899-924, https:// doi.org/10.1002/qj.49712656407.

Fedorov, A. V., and S. G. Philander, 2000: Is El Niño changing? Science, 288, 1997-2002, https://doi.org/10.1126/science.288. 5473.1997.

— S. Hu, M. Lengaigne, and E. Guilyardi, 2015: The impact of westerly wind bursts and ocean initial state on the development, and diversity of El Niño events. Climate Dyn., 44, 13811401, https://doi.org/10.1007/s00382-014-2126-4.

Feng, J., and T. Lian, 2018: Assessing the relationship between MJO and equatorial Pacific WWBs in observations and CMIP5 models. J. Climate, 31, 6393-6410, https://doi.org/ 10.1175/JCLI-D-17-0526.1.

Freund, M. B., B. J. Henley, D. J. Karoly, H. V. McGregor, N. J. Abram, and D. Dommenget, 2019: Higher frequency of central Pacific El Niño events in recent decades relative to past centuries. Nat. Geosci., 12, 450-455, https://doi.org/10.1038/ s41561-019-0353-3.
Gao, R., R. Zhang, M. Wen, and T. Li, 2019: Interdecadal changes in the asymmetric impacts of ENSO on wintertime rainfall over China and atmospheric circulations over western North Pacific. Climate Dyn., 52, 7525-7536, https://doi.org/10.1007/ s00382-018-4282-4.

Hendon, H. H., M. C. Wheeler, and C. Zhang, 2007: Seasonal dependence of the MJO-ENSO relationship. J. Climate, 20, 531543, https://doi.org/10.1175/JCLI4003.1.

$\mathrm{Hu}, \mathrm{S}$, and A. V. Fedorov, 2016: Exceptionally strong easterly wind burst stalling El Niño of 2014. Proc. Natl. Acad. Sci. USA, 113, 2005-2010, https://doi.org/10.1073/pnas.1514182113.

—_, and — 2019: The extreme El Niño of 2015-2016: The role of westerly and easterly wind bursts, and preconditioning by the failed 2014 event. Climate Dyn., 52, 7339-7357, https:// doi.org/10.1007/s00382-017-3531-2.

,$- \ldots$, M. Lengaigne, and E. Guilyardi, 2014: The impact of westerly wind bursts on the diversity and predictability of $\mathrm{El}$ Niño events: An ocean energetics perspective. Geophys. Res. Lett., 41, 4654-4663, https://doi.org/10.1002/2014GL059573.

Imada, Y., and M. Kimoto, 2009: ENSO amplitude modulation related to Pacific decadal variability. Geophys. Res. Lett., 36, L03706, https://doi.org/10.1029/2008GL036421.

Jin, E. K., and Coauthors, 2008: Current status of ENSO prediction skill in coupled ocean-atmosphere models. Climate Dyn., 31, 647-664, https://doi.org/10.1007/s00382-008-0397-3.

Jin, F.-F., 1997a: An equatorial ocean recharge paradigm for ENSO. Part I: Conceptual model. J. Atmos. Sci., 54, 811-829, https://doi.org/10.1175/1520-0469(1997)054<0811: AEORPF $>2.0 . \mathrm{CO} ; 2$.

- 1997b: An equatorial ocean recharge paradigm for ENSO. Part II: A stripped-down coupled model. J. Atmos. Sci., 54, 830-847, https://doi.org/10.1175/1520-0469(1997)054<0830: AEORPF $>2.0 . \mathrm{CO} ; 2$.

, and S.-I. An, 1999: Thermocline and zonal advective feedbacks within the equatorial ocean recharge oscillator model for ENSO. Geophys. Res. Lett., 26, 2989-2992, https://doi.org/ 10.1029/1999GL002297.

— S. T. Kim, and L. Bejarano, 2006: A coupled-stability index for ENSO. Geophys. Res. Lett., 33, L23708, https://doi.org/ 10.1029/2006GL027221.

Jones, C., D. E. Waliser, K. Lau, and W. Stern, 2004: Global occurrences of extreme precipitation and the Madden-Julian oscillation: Observations and predictability. J. Climate, 17, 4575-4589, https://doi.org/10.1175/3238.1.

Kirtman, B., 2019: ENSO diversity. Climate Dyn., 52, 7133, https:// doi.org/10.1007/s00382-019-04723-2.

Knutson, T. R., S. Manabe, and D. Gu, 1997: Simulated ENSO in a global coupled ocean-atmosphere model: Multidecadal amplitude modulation and $\mathrm{CO}_{2}$ sensitivity. J. Climate, 10, 138-161, https://doi.org/10.1175/1520-0442(1997)010<0138: SEIAGC $>2.0 . \mathrm{CO} ; 2$.

Kug, J.-S., F.-F. Jin, and S.-I. An, 2009: Two types of El Niño events: Cold tongue El Niño and warm pool El Niño. J. Climate, 22, 1499-1515, https://doi.org/10.1175/2008JCLI2624.1.

Larson, S., and B. Kirtman, 2013: The Pacific meridional mode as a trigger for ENSO in a high-resolution coupled model. Geophys. Res. Lett., 40, 3189-3194, https://doi.org/10.1002/grl.50571.

Lemmon, D. E., and K. B. Karnauskas, 2019: A metric for quantifying El Niño pattern diversity with implications for ENSOmean state interaction. Climate Dyn., 52, 7511-7523, https:// doi.org/10.1007/s00382-018-4194-3.

Lengaigne, M., E. Guilyardi, J.-P. Boulanger, C. Menkes, P. Delecluse, P. Inness, J. Cole, and J. Slingo, 2004: 
Triggering of El Niño by westerly wind events in a coupled general circulation model. Climate Dyn., 23, 601-620, https:// doi.org/10.1007/s00382-004-0457-2.

LeVeque, R. J., and Coauthors, 2002: Finite Volume Methods for Hyperbolic Problems. Vol. 31, Cambridge University Press, $558 \mathrm{pp}$.

Levine, A. F., and M. J. McPhaden, 2016: How the July 2014 easterly wind burst gave the 2015-2016 El Niño a head start. Geophys. Res. Lett., 43, 6503-6510, https://doi.org/10.1002/ 2016 GL069204.

L'Heureux, M. L., S. Lee, and B. Lyon, 2013: Recent multidecadal strengthening of the Walker circulation across the tropical Pacific. Nat. Climate Change, 3, 571-576, https://doi.org/10.1038/ nclimate1840.

Lian, T., D. Chen, Y. Tang, and Q. Wu, 2014: Effects of westerly wind bursts on El Niño: A new perspective. Geophys. Res. Lett., 41, 3522-3527, https://doi.org/10.1002/ 2014GL059989.

Majda, A., 2003: Introduction to PDEs and Waves for the Atmosphere and Ocean. Courant Lecture Notes in Mathematics, Vol. 9, American Mathematical Society, 234 pp.

- , and S. N. Stechmann, 2009: The skeleton of tropical intraseasonal oscillations. Proc. Natl. Acad. Sci. USA, 106, 8417 8422, https://doi.org/10.1073/pnas.0903367106.

McPhaden, M. J., S. E. Zebiak, and M. H. Glantz, 2006a: ENSO as an integrating concept in Earth science. Science, 314, 17401745, https://doi.org/10.1126/science.1132588.

—, X. Zhang, H. H. Hendon, and M. C. Wheeler, 2006b: Large scale dynamics and MJO forcing of ENSO variability. Geophys. Res. Lett., 33, L16702, https://doi.org/10.1029/ 2006 GL026786.

Meng, Q., M. Latif, W. Park, N. S. Keenlyside, V. A. Semenov, and T. Martin, 2012: Twentieth century Walker circulation change: Data analysis and model experiments. Climate Dyn., 38, 17571773, https://doi.org/10.1007/s00382-011-1047-8.

Nakazawa, T., 1988: Tropical super clusters within intraseasonal variations over the western Pacific. J. Meteor. Soc. Japan, 66, 823-839, https://doi.org/10.2151/jmsj1965.66.6_823.

Puy, M., J. Vialard, M. Lengaigne, and E. Guilyardi, 2016: Modulation of equatorial Pacific westerly/easterly wind events by the Madden-Julian oscillation and convectively-coupled Rossby waves. Climate Dyn., 46, 2155-2178, https://doi.org/ 10.1007/s00382-015-2695-x.

— stochasticity on El Niño amplitude: The case of 2014 vs. 2015. Climate Dyn., 52, 7435-7454, https://doi.org/10.1007/s00382017-3938-9.

—, J. Vialard, M. Lengaigne, E. Guilyardi, A. Voldoire, and G. Madec, 2019b: Modulation of equatorial Pacific sea surface temperature response to westerly wind events by the oceanic background state. Climate Dyn., 52, 7267-7291, https://doi.org/ 10.1007/s00382-016-3480-1.

Rayner, N., D. E. Parker, E. Horton, C. K. Folland, L. V. Alexander, D. Rowell, E. Kent, and A. Kaplan, 2003: Global analyses of sea surface temperature, sea ice, and night marine air temperature since the late nineteenth century. J. Geophys. Res., 108, 4407, https://doi.org/10.1029/ 2002JD002670.

Ren, H.-L., and F.-F. Jin, 2013: Recharge oscillator mechanisms in two types of ENSO. J. Climate, 26, 6506-6523, https://doi.org/ 10.1175/JCLI-D-12-00601.1.

Reynolds, R. W., T. M. Smith, C. Liu, D. B. Chelton, K. S. Casey, and M. G. Schlax, 2007: Daily high-resolution-blended ana- lyses for sea surface temperature. J. Climate, 20, 5473-5496, https://doi.org/10.1175/2007JCLI1824.1.

Schopf, P. S., 1987: Coupled dynamics of the tropical ocean-atmosphere system. Further Progress in Equatorial Oceanography, Nova University Press, 279-86.

—_, and M. J. Suarez, 1988: Vacillations in a coupled oceanatmosphere model. J. Atmos. Sci., 45, 549-566, https://doi.org/ 10.1175/1520-0469(1988)045<0549:VIACOM>2.0.CO;2.

Seiki, A., and Y. N. Takayabu, 2007a: Westerly wind bursts and their relationship with intraseasonal variations and ENSO. Part I: Statistics. Mon. Wea. Rev., 135, 3325-3345, https:// doi.org/10.1175/MWR3477.1.

— with intraseasonal variations and ENSO. Part II: Energetics over the western and central Pacific. Mon. Wea. Rev., 135, 3346-3361, https://doi.org/10.1175/MWR3503.1.

Stevenson, S., A. Capotondi, J. Fasullo, and B. Otto-Bliesner, 2019: Forced changes to twentieth century ENSO diversity in a last millennium context. Climate Dyn., 52, 7359-7374, https:// doi.org/10.1007/s00382-017-3573-5.

Su, J., R. Zhang, T. Li, X. Rong, J. Kug, and C.-C. Hong, 2010: Causes of the El Niño and La Niña amplitude asymmetry in the equatorial eastern Pacific. J. Climate, 23, 605-617, https:// doi.org/10.1175/2009JCLI2894.1.

Suarez, M. J., and P. S. Schopf, 1988: A delayed action oscillator for ENSO. J. Atmos. Sci., 45, 3283-3287, https://doi.org/10.1175/ 1520-0469(1988)045<3283:ADAOFE>2.0.CO;2.

Tam, C.-y., and N.-C. Lau, 2005: Modulation of the Madden-Julian oscillation by ENSO: Inferences from observations and GCM simulations. J. Meteor. Soc. Japan, 83, 727-743, https://doi.org/ 10.2151/jmsj.83.727.

Tang, Y., and B. Yu, 2008: MJO and its relationship to ENSO. J. Geophys. Res., 113, D14106, https://doi.org/10.1029/ 2007JD009230.

— predictability study. Natl. Sci. Rev., 5, 826-839, https://doi.org/ 10.1093/nsr/nwy105.

Taschetto, A. S., A. S. Gupta, N. C. Jourdain, A. Santoso, C. C. Ummenhofer, and M. H. England, 2014: Cold tongue and warm pool ENSO events in CMIP5: Mean state and future projections. J. Climate, 27, 2861-2885, https://doi.org/10.1175/JCLI-D13-00437.1.

Thual, S., A. J. Majda, and S. N. Stechmann, 2014: A stochastic skeleton model for the MJO. J. Atmos. Sci., 71, 697-715, https://doi.org/10.1175/JAS-D-13-0186.1.

,-- , and -2015 : Asymmetric intraseasonal events in the stochastic skeleton MJO model with seasonal cycle. Climate Dyn., 45, 603-618, https://doi.org/10.1007/s00382014-2256-8.

,,-- N. Chen, and S. N. Stechmann, 2016: Simple stochastic model for El Niño with westerly wind bursts. Proc. Natl. Acad. Sci. USA, 113, $10245-10250$, https://doi.org/10.1073/ pnas.1612002113.

-, , and,- 2017 : Seasonal synchronization of a simple stochastic dynamical model capturing El Niño diversity. J. Climate, 30, 10 047-10 066, https://doi.org/10.1175/JCLI-D17-0174.1.

,-- , and —_ 2018: A tropical stochastic skeleton model for the MJO, El Niño, and dynamic Walker circulation: A simplified GCM. J. Climate, 31, 9261-9282, https://doi.org/ 10.1175/JCLI-D-18-0263.1.

,-- , and -2019 : Statistical occurrence and mechanisms of the 2014-2016 delayed super El Niño captured by a simple 
dynamical model. Climate Dyn., 52, 2351-2366, https://doi.org/ 10.1007/s00382-018-4265-5.

Timmermann, A., and Coauthors, 2018: El Niño-Southern Oscillation complexity. Nature, 559, 535-545, https://doi.org/10.1038/s41586018-0252-6.

Vecchi, G. A., 2006: The termination of the 1997-98 El Niño. Part II: Mechanisms of atmospheric change. J. Climate, 19, 26472664, https://doi.org/10.1175/JCLI3780.1.

Wang, C., and J. Picaut, 2004: Understanding ENSO physicsA review. Earth's Climate: The Ocean-Atmosphere Interaction, Geophys. Monogr., Vol. 147, Amer. Geophys. Union, 21-48.

Yeh, S.-W., and Coauthors, 2018: ENSO atmospheric teleconnections and their response to greenhouse gas forcing. Rev. Geophys., 56, 185-206, https://doi.org/10.1002/2017RG000568.

Yu, J.-Y., and S. T. Kim, 2011: Relationships between extratropical sea level pressure variations and the central Pacific and east- ern Pacific types of ENSO. J. Climate, 24, 708-720, https:// doi.org/10.1175/2010JCLI3688.1.

Zebiak, S. E., 1986: Atmospheric convergence feedback in a simple model for El Niño. Mon. Wea. Rev., 114, 1263-1271, https:// doi.org/10.1175/1520-0493(1986)114<1263:ACFIAS>2.0.CO;2. 1989: Oceanic heat content variability and El Niño cycles. J. Phys. Oceanogr., 19, 475-486, https://doi.org/10.1175/15200485(1989)019<0475:OHCVAE > 2.0.CO;2.

— , and M. A. Cane, 1987: A model El Niño-Southern Oscillation. Mon. Wea. Rev., 115, 2262-2278, https://doi.org/10.1175/15200493(1987)115<2262:AMENO>2.0.CO;2.

Zhang, C., 2005: Madden-Julian oscillation. Rev. Geophys., 43, RG2003, https://doi.org/10.1029/2004RG000158.

Zhang, T., and D.-Z. Sun, 2014: ENSO asymmetry in CMIP5 models. J. Climate, 27, 4070-4093, https://doi.org/10.1175/ JCLI-D-13-00454.1. 